\title{
Compartmentalized Regulation of Parkin-Mediated Mitochondrial Quality Control in the Drosophila Nervous System In Vivo
}

\author{
Hyun Sung, Lauren C. Tandarich, Kenny Nguyen, and Peter J. Hollenbeck \\ Department of Biological Sciences, Purdue University, West Lafayette, Indiana 47907
}

In neurons, the normal distribution and selective removal of mitochondria are considered essential for maintaining the functions of the large asymmetric cell and its diverse compartments. Parkin, a E3 ubiquitin ligase associated with familial Parkinson's disease, has been implicated in mitochondrial dynamics and removal in cells including neurons. However, it is not clear how Parkin functions in mitochondrial turnover in vivo, or whether Parkin-dependent events of the mitochondrial life cycle occur in all neuronal compartments. Here, using the live Drosophila nervous system, we investigated the involvement of Parkin in mitochondrial dynamics, distribution, morphology, and removal. Contrary to our expectations, we found that Parkin-deficient animals do not accumulate senescent mitochondria in their motor axons or neuromuscular junctions; instead, they contain far fewer axonal mitochondria, and these displayed normal motility behavior, morphology, and metabolic state. However, the loss of Parkin did produce abnormal tubular and reticular mitochondria restricted to the motor cell bodies. In addition, in contrast to drug-treated, immortalized cells in vitro, mature motor neurons rarely displayed Parkin-dependent mitophagy. These data indicate that the cell body is the focus of Parkin-dependent mitochondrial quality control in neurons, and argue that a selection process allows only healthy mitochondria to pass from cell bodies to axons, perhaps to limit the impact of mitochondrial dysfunction.

Key words: autophagy; axonal transport; Drosophila; mitochondria; mitophagy; Parkin

\section{Significance Statement}

Parkin has been proposed to police mitochondrial fidelity by binding to dysfunctional mitochondria via PTEN (phosphatase and tensin homolog)-induced putative kinase 1 (PINK1) and targeting them for autophagic degradation. However, it is unknown whether and how the PINK1/Parkin pathway regulates the mitochondrial life cycle in neurons in vivo. Using Drosophila motor neurons, we show that parkin disruption generates an abnormal mitochondrial network in cell bodies in vivo and reduces the number of axonal mitochondria without producing any defects in their axonal transport, morphology, or metabolic state. Furthermore, while cultured neurons display Parkin-dependent axonal mitophagy, we find this is vanishingly rare in vivo under normal physiological conditions. Thus, both the spatial distribution and mechanism of mitochondrial quality control in vivo differ substantially from those observed in vitro.

\section{Introduction}

Neurons are large, morphologically asymmetric and functionally compartmentalized cells. For this reason, they rely critically on

\footnotetext{
Received Feb. 26, 2016; revised April 7, 2016; accepted May 18, 2016.

Author contributions: H.S. and P.J.H. designed research; H.S. performed research; H.S., L.C.T., and K.N. analyzed data; H.S. and P.J.H. wrote the paper.

This work was supported by National Institutes of Health/National Institute of Neurological Disorders and Stroke Grant 5R01-NS-027073. We thank L.J. Pallanck (University of Washington, Seattle, WA) for providing flies (D42Gal4>UAS-mitoGFP), (park ${ }^{25}$, D42-Gal4/TM6B), (park ${ }^{25}$, UAS-mitoGFP/TM6B), and (UAS-Parkin/Cy0); E. Hafen (ETH, Zürich, Switzerland) for providing flies (UAS-RFPatg8/Cy0); J.C. Clemens (Purdue University, West Lafayette, IN) for providing anti-HRP; and Elisabeth Garland-Kuntz for assisting with blinded analysis of mitochondrial morphology.

The authors declare no competing financial interests.

Correspondence should be addressed to Peter J. Hollenbeck, Department of Biological Sciences, Purdue University, 915 West State Street, West Lafayette, IN 47907. E-mail: phollenb@purdue.edu.
}

robust, organized axonal transport to maintain the normal distribution of organelles among the somatodendritic, axonal, and synaptic compartments (Hirokawa et al., 2010; Saxton and Hollenbeck, 2012). Mitochondria play an important role in neuronal function and survival, as they supply ATP, buffer cytosolic calcium, and generate reactive oxygen species, and thus their transport and redistribution within neurons are particularly important (Saxton and Hollenbeck, 2012). They display a unique and complex life cycle that is characterized by bidirectional movement (Stowers et al., 2002; Guo et al., 2005; Glater et al., 2006; Pilling et al., 2006; Reis et al., 2009), morphological changes 
(Okamoto and Shaw, 2005; Chan, 2006; Song et al., 2009), and complex biogenesis and degradation (Davis and Clayton, 1996; Amiri and Hollenbeck, 2008; Maday et al., 2012; Ashrafi et al., 2014). In neurons, these components of the mitochondrial life cycle are closely interrelated: for example, the dysregulation of mitochondrial axonal transport can affect organelle morphology (Pathak et al., 2010), while altered fission-fusion balance can impair axonal mitochondrial motility (Verstreken et al., 2005; Baloh et al., 2007; Misko et al., 2010; Yu et al., 2016). Although it has been thought that the neuronal lysosomal compartment resides mainly near the nucleus, the presence, transport, and development of late endosomes (Deinhardt et al., 2006) and lysosomes (Moughamian and Holzbaur, 2012) have been demonstrated in axons. Additionally, in axons, local biogenesis (Amiri and Hollenbeck, 2008), fission-fusion (Cagalinec et al., 2013), and turnover (Maday et al., 2012; Ashrafi et al., 2014) of mitochondria are reported to occur. Thus, the regulation of the mitochondrial life cycle and the maintenance of a robust functional population, collectively termed mitochondrial "quality control" (QC; Chen and Chan, 2009; Rugarli and Langer, 2012), are likely to be more complex in neurons in vivo than in cells of more modest dimensions due to the potential distribution of functions among distant compartments.

It has been proposed that mitochondrial dynamics and $\mathrm{QC}$ are functionally regulated by two Parkinson's disease-related genes, PTEN (phosphatase and tensin homolog)-induced putative kinase 1 (PINK1) and the E3 ubiquitin ligase Parkin. Recent data from Drosophila suggest that PINK1/Parkin catalyze mitochondrial arrest by tagging for degradation of the mitochondriakinesin linker protein Miro (Wang et al., 2011; Liu et al., 2012), and also prevent the fusion of senescent mitochondria with healthy ones by downregulating the mitochondrial fusion protein Mitofusin 2 (Deng et al., 2008; Poole et al., 2008, 2010). Furthermore, studies in non-neuronal cells indicate that PINK1 and Parkin target depolarized, dysfunctional mitochondria for autophagic engulfment and degradation (Narendra et al., 2008, 2010; Matsuda et al., 2010; Ashrafi et al., 2014; Lazarou et al., 2015). Although the question of whether this pathway governs mitochondrial QC in all neuronal compartments remains unsolved, these findings suggest that PINK1 and Parkin may cooperate in the maintenance of neuronal mitochondrial distribution and function by regulating mitochondrial dynamics and turnover.

Here, we used the intact Drosophila larval nervous system for in vivo study, complemented by cultured larval primary neurons as an in vitro system, to analyze the life cycle of neuronal mitochondria. We assessed the following three processes: mitochondrial axonal transport, fission-fusion, and degradation. We found that the loss of Parkin diminishes axonal mitochondrial flux without disturbing most features of movement. In addition, although Parkin-deficient motor neurons displayed greatly reduced numbers of axonal mitochondria, their organelle morphology and membrane potential were normal. However, mitochondrial morphology was disrupted in cell bodies, suggesting that this compartment houses Parkin-dependent QC in vivo. We also observed a striking difference in the extent of axonal mitophagy between in vitro motor neurons, where it was abundant, and the same neurons in vivo, where it was rare. Together, these observations indicate that Parkin-dependent mitochondrial QC differs significantly in location and abundance between in vivo and in vitro systems, and further suggest that Parkin regulates mitochondrial axonal access in vivo.

\section{Materials and Methods}

Drosophila strains and culture conditions. All flies were reared on standard cornmeal agar medium and maintained at $25^{\circ} \mathrm{C}$ with a $12 \mathrm{~h} \mathrm{light/}$ dark cycle. The GAL4-UAS system was used to visualize mitochondria, and autophagosomes specifically, in motor neurons. Wild-type flies expressing a single copy of mitochondrially targeted GFP (mito-GFP) or coexpressing mito-GFP with RFP-Atg 8 driven by $D 42$ driver were used as the controls $(w ;+/+$; D42-Gal4>UAS-mitoGFP/+) and $(w$; UAS-RFPatg8/UAS-RFPatg8; D42-Gal4>UAS-mitoGFP/+; Pilling et al., 2006). Parkin-null mutants, $\left(w\right.$; +/+; park $^{25}$, D42-Gal4/park ${ }^{25}$, UAS-mitoGFP) and (w; UAS-RFPatg8/UAS-RFPatg8; park ${ }^{25}$, D42-Gal4/park ${ }^{25}$, UASmitoGFP), were used in this study (Greene et al., 2003). Genetically overexpressed Parkin in the null mutant background, ( $w$; UAS-Parkin/+; park $^{25}$, D42-Gal4/park ${ }^{25}$, UAS-mitoGFP), was used for rescue experiments. For starvation conditions, third instar larvae were collected and maintained in a distilled water-moisturized dish without food for $6 \mathrm{~h}$.

Dissected larval preparation for observing neuronal mitochondria in vivo. To image mitochondria in live motor neurons, late third instar larvae were collected, dissected, and prepared as previously described (Devireddy et al., 2014). In brief, larvae were pinned ventral side down on a Sylgard plate and dissected with a handheld scissors (catalog \#15000-00, Fine Science Tools) from posterior to anterior along the dorsal body wall in HL6 buffer containing $0.6 \mathrm{~mm} \mathrm{CaCl}_{2}$ and $4 \mathrm{~mm}$ [SCAP] L-glutamate. Fat bodies and intestine were then removed to reveal the intact ventral ganglion (VG), segmental nerves (SNs), and neuromuscular junctions (NMJs; Pilling et al., 2006; Louie et al., 2008; Shidara and Hollenbeck, 2010). The dissected larva was then placed dorsal side up on a glass slide and covered by a glass coverslip using dental wax as a spacer to form a chamber. This was filled with HL6 buffer and sealed with Valap for imaging. Mitochondria in both cell bodies and axons of larval motor neurons were observed using laser-scanning confocal microscopy (LSCM; C1 on an Eclipse 90i microscope, Nikon) within $20 \mathrm{~min}$ of dissection to ensure normal in vivo conditions of the nervous system (Shidara and Hollenbeck, 2010; Devireddy et al., 2014).

Time-lapse microscopy for organelle axonal transport analysis. To analyze axonal transport of organelle in motor neurons, time-lapse images of mito-GFP and RFP-Atg8 were acquired from the longest SN in the following three different regions: proximal (segment A2); middle (segment A4); and distal (segment A7) to the VG. Time-lapse confocal images were obtained using $5 \%$ laser power with a $488 \mathrm{~nm}$ band for mito-GFP and $10 \%$ laser power with a $561 \mathrm{~nm}$ band for RFP-Atg 8 through the smallest pinhole $(30 \mu \mathrm{m})$. The region imaged was $50 \mu \mathrm{m}$ in length, and frames for individual channels (513/30 for mito-GFP and 590/50 for RFP-Atg8) were obtained at $1 \mathrm{~s}$ time intervals for $2 \mathrm{~min}$. The following parameters of transport were quantified essentially as previously described by the manual tracking function of ImageJ software (Devireddy et al., 2014). Briefly, mitochondrial and autophagosomal flux were measured for both anterograde and retrograde movement by observing the number of moving organelles passing a defined point per unit time over $2 \mathrm{~min}$. Since some diffuse RFP-Atg8 signals were seen throughout the cytosol, only vacuoles above a threshold intensity ( 1000 on a 12 bit scale) were designated as autophagic vacuoles (AVs). Mitochondrial velocity was measured from continuous mitochondrial movements of $>3 \mathrm{~s}$ duration in one direction. One pixel represents $0.1 \mu \mathrm{m}$ in our confocal images, and only net velocities of $>0.1 \mu \mathrm{m} / \mathrm{s}$ or $<-0.1 \mu \mathrm{m} / \mathrm{s}$ for at least three consecutive frames were selected as bona fide anterograde and retrograde transport, respectively. During 2 min time-lapse movies, only mitochondria that moved for $>60 \mathrm{~s}$ were considered for velocity analysis. Mitochondrial moving and stationary percentage was measured from the total population of mitochondria within a field. Since the population of mitochondria within the assigned axonal regions included a changing set of moving mitochondria, the second frame of each movie was selected for designating mitochondrial total population. Mitochondria in the population were categorized and marked as anterograde, retrograde, or stationary by ImageJ/Cell Counter to quantify the percentage of moving and stationary mitochondria in segmental axons. The mitochondrial duty cycle was measured for moving mitochondria by observing their movement or pause time over the observation interval. The percentage of the time that 
mitochondria moved in each particular direction or paused was quantified, and again only mitochondria that moved for $>60$ s were considered for duty cycles. Mitochondrial run length was defined as the distance per individual run between stops. Mitochondrial net runs in the dominant direction only for both anterograde and retrograde movement were used for the run length measurement. Mitochondrial flux with photobleaching was measured from $50 \mu \mathrm{m}$ regional length of middle (segment A4) SNs with $30 \mu \mathrm{m}$ photo bleached for $30 \mathrm{~s}$ with full intensity of $488 \mathrm{~nm}$ light from the confocal laser. Time course images were acquired at a rate of 1 frame every $2 \mathrm{~s}$ for $5 \mathrm{~min}$. Every $30 \mathrm{~s}$, the number of steady-state moving mitochondria was quantified from the bleached regions. An exponential series was applied to estimate the number of steady-state moving mitochondria in bleached regions. Thirty and 29 steady-state moving mitochondria were estimated in control and park ${ }^{25}$, Parkin animals respectively, while 17 steady-state moving mitochondria were estimated in park ${ }^{25}$ mutants from the $30 \mu \mathrm{m}$ photo-bleached regions, as follows: control from $\left(y=0.033 \mathrm{e}^{63.612 \mathrm{x}}\right)$, park ${ }^{25}$ from $\left(y=0.059 \mathrm{e}^{58.643 \mathrm{x}}\right)$, and park $^{25}$, Parkin from $\left(y=0.035 \mathrm{e}^{63.741 \mathrm{x}}\right)$. These estimated numbers were nearly consistent with the numbers calculated from the percentage of moving mitochondria, as follows: $\sim 27$ steady-state moving mitochondria were calculated in controls, while $\sim 16$ steady-state moving mitochondria were calculated in park $^{25}$ mutants from the bleached regions (data not shown).

Determination of organelle density and colocalization. MetaMorph version 7.6.5 software was used to measure the mitochondrial density in SNs based on the intensity of the mito-GFP signal (Devireddy et al., 2014). The second frame of each movie was selected, and the number of pixels with GFP signal above threshold was used to determine the density of mitochondria in the region of observation. To clarify the shape of the mitochondrial boundary, the selected frames of confocal images are converted to binary images with 400 minimum-intensity thresholds on a 12 bit scale of $0-4095$. Mitochondrial density in NMJs was measured by NIS-Elements AR 3.2 software to analyze the area. In each NMJ, the polygonal ROI tool was used to draw the region around the synaptic boutons, and the area that was covered by mitochondria was calculated with the total area. A 515/30 (mito-GFP) channel was used for measuring mitochondrial density, whereas a 590/50 (anti-HRP) channel was used for measuring the area of synaptic boutons. The images were converted by representative thresholds ( 400 minimum intensity for mitochondria, and 200 minimum intensity for anti-HRP staining) to clarify the shape of the boundaries. Colocalization of AVs with mitochondria was quantified by NIS-Elements AR 3.2 software. The intensity profiles from two channels, 515/30 (mito-GFP) and 590/50 (RFP-Atg8), were used to analyze the colocalization in each region.

Immunostaining. For immunostaining of NMJs, late third instar larvae were partially dissected and fixed with $4 \%$ paraformaldehyde for $20 \mathrm{~min}$ at room temperature (Shidara and Hollenbeck, 2010). Fixed samples were washed with PBT (PBS containing $0.1 \%$ Triton X-100), and blocked with PBTB (PBT containing $0.2 \%$ BSA), then stained with mouse antiHRP (1:1000) that was conjugated with Alexa Fluor 594 goat anti-mouse (a gift from J.C. Clemens, Purdue University, West Lafayette, IN). Immunostained samples were washed with PBTB before mounting and were observed by LSCM (C1 on an Eclipse 90i microscope, Nikon). Images of immunostained NMJs were taken between muscles 6 and 7 from A4 SNs.

Axonal mitochondria in adult fly wing. To observe axonal mitochondria in the wing nerve, 5-d-old adult flies were collected and paralyzed on $\mathrm{a} \mathrm{CO}_{2}$ anesthetizing pad. The fly wings were visualized under a dissection stereomicroscope and cut at the end of wing root to preserve a whole nerve tract along the humeral crossvein ( $\mathrm{HCV}$ ) with the first lateral vein (LV1) and costal vein of LV0 (Fang et al., 2012). Severed wings were pretreated with detergent $\left(20 \%\right.$, Triton $\mathrm{X}-100$ in $\left.\mathrm{dH}_{2} \mathrm{O}\right)$ to avoid trapping air bubbles, briefly rinsed with $1 \times$ PBS (Fang et al., 2013), placed dorsal side up on a glass slide, and covered by a glass coverslip using dental wax as a spacer. The chamber was filled by PBS and sealed with Valap for imaging. Images were taken using LSCM within $10 \mathrm{~min}$ to ensure normal in vivo conditions of the wing neuronal system. Axons of $\mathrm{HCV}$ from the wing arch were monitored to analyze axonal mitochon- drial density. In all experiments, only the right wing from the female adult was used.

Morphological analysis of mitochondria. Image quantification was performed using NIS-Elements AR 3.2 software. Nonoverlapping axonal mitochondria from each SN (segment A2, segment A4, and segment A7) were selected for both length and area measurements, while in primary cultured neurons only length measurements were performed. Since 1 pixel represents $0.1 \mu \mathrm{m}$ in the NIS-Elements AR 3.2 software images, the minimum standard distance unit was $0.1 \mu \mathrm{m}$. To determine the boundary of mitochondria for the length and area measurements, images were thresholded at a standard intensity of 200 to identify pixels belonging to mitochondria. Mitochondrial morphology in cell bodies was analyzed using both individual sections and three-dimensional image stacks. The latter were built from $20 \mu \mathrm{m}$ (X, height) by $20 \mu \mathrm{m}$ (Y, width) with $5 \mu \mathrm{m}$ ( $Z$, depth) of $100 z$-stacks centered on the middle of the cell body. Morphological analyses were performed blinded to the experimental condition.

Measurement for mitochondrial membrane potential. Tetramethylrhodamine methyl ester (TMRM; Life Technologies), a lipophilic cationic fluorescent dye, was used for the determination of mitochondrial transmembrane potential both in vivo (Devireddy et al., 2014) and in vitro (Verburg and Hollenbeck, 2008). Thin neurons and NMJs adjacent to the middle SNs in larval A4 segment and projected axons of primary cells were selected for measuring neuronal mitochondrial membrane potential $\left(\Delta \Psi_{\mathrm{m}}\right)$ in in vivo and in vitro conditions, respectively. TMRM $200 \mathrm{nM}$ in prepared medium (HL6 buffer or Schneider's medium) was added to the dissected larvae or cultured cells for $20 \mathrm{~min}$, then replaced with $50 \mathrm{~nm}$ TMRM for imaging. Only nonoverlapping axonal mitochondria were considered for quantification. Images were obtained sequentially by 488 $\mathrm{nm}$ excitation with $5 \%$ laser power for mito-GFP and by $561 \mathrm{~nm}$ excitation with 5\% laser power for TMRM. The ratio of mitochondrial fluorescence intensities $\left(\mathrm{F}_{\mathrm{m}}\right)$ to cytoplasmic fluorescence intensities $\left(\mathrm{F}_{\mathrm{c}}\right)$ was used to determine $\Delta \Psi_{\mathrm{m}}\left(\mathrm{F}_{\mathrm{m}} / \mathrm{F}_{\mathrm{c}} ;\right.$ Verburg and Hollenbeck, 2008; Shidara and Hollenbeck, 2010; Devireddy et al., 2014).

Primary neuronal cell culture. Brain lobes and ventral ganglia were taken from third instar larvae and transferred into Schneider's medium. Tissue was incubated in $0.7 \mathrm{mg} / \mathrm{ml}$ collagenase for $1 \mathrm{~h}$ at room temperature and then dissociated to individual cells by siliconized pipette triturating. After dissociation, $\sim 250 \mu \mathrm{l}$ of cell solution was transferred to each glass coverslip in a cell culture dish and incubated for $1 \mathrm{~h}$ for cell adhesion. Glass coverslips were coated with $20 \mu \mathrm{g} / \mathrm{ml}$ concanavalin A for cell adhesion. Five to seven sets of CNS were used for two $35 \mathrm{~mm}$ cell culture dishes. After $1 \mathrm{~h}$ of incubation, $2 \mathrm{ml}$ of Schneider's medium was added, and culture dishes were incubated for $\sim 96 \mathrm{~h}$ to allow neurons to extend their processes. Antimycin A (Ant A; $5 \mu \mathrm{M}$ ) was treated for $20 \mathrm{~min}$ to depolarize mitochondria. For oxidative stress conditions, $100 \mu \mathrm{M} \mathrm{H}_{2} \mathrm{O}_{2}$ was added to the culture dishes $1 \mathrm{~h}$ before taking images.

Statistical analysis. To analyze data from park ${ }^{25}$ mutants compared with controls, $p$ values were calculated using an unpaired homoscedastic $t$ test. For multiple group comparisons, statistical significance was calculated by a one-way ANOVA with Bonferroni correction post hoc test. Significance between two populations was determined by two-sample Kolmogorov-Smirnov (K-S) test for analyzing the critical distribution. Correlations between two parameters were assessed by Pearson's correlation coefficient $R^{2}$ values. In all cases, at least three independent experiments were performed. All statistical significance was verified at ${ }^{*} p<$ $0.05,{ }^{* *} p<0.01$, and ${ }^{* *} p<0.001$ using GraphPad Prism.

\section{Results}

\section{Parkin loss affects axonal mitochondrial flux without disturbing most features of movement}

The PINK1/Parkin pathway has been proposed both to support mitochondrial QC and turnover (Narendra et al., 2008, 2010; Matsuda et al., 2010), and to regulate mitochondrial motility (Wang et al., 2011; Liu et al., 2012; Saxton and Hollenbeck, 2012). These two functions are likely to be related, as the return of senescent mitochondria to the cell body would require targeted net retrograde traffic. Thus, one expectation is that Parkin deficiency 
A

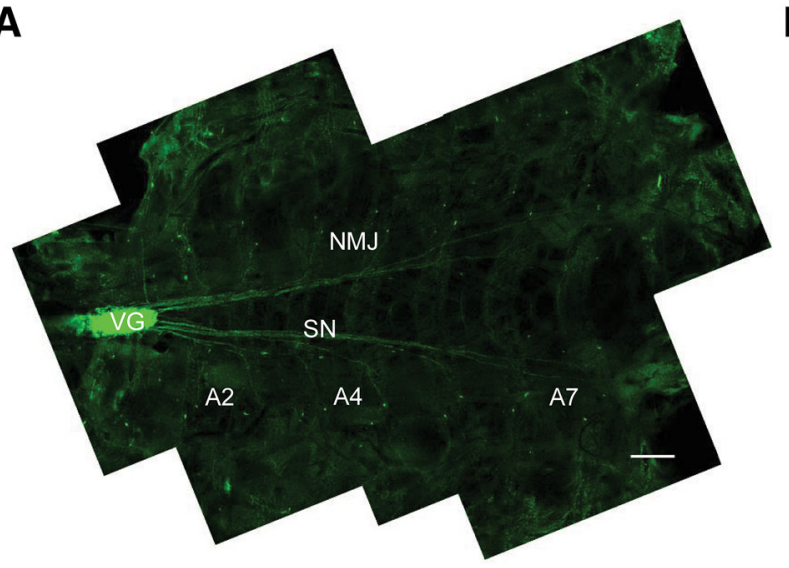

C
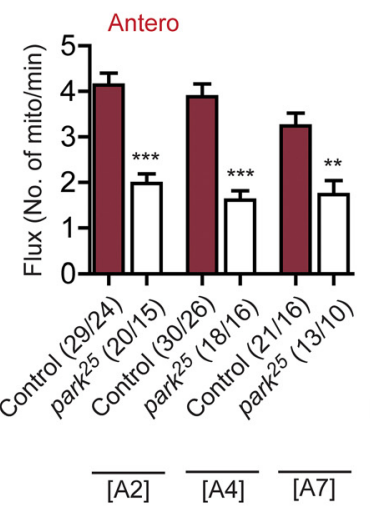

$\mathbf{E}$
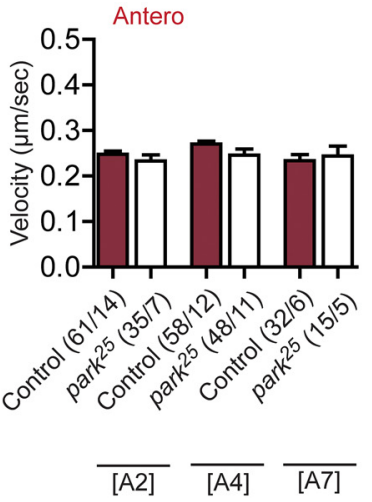
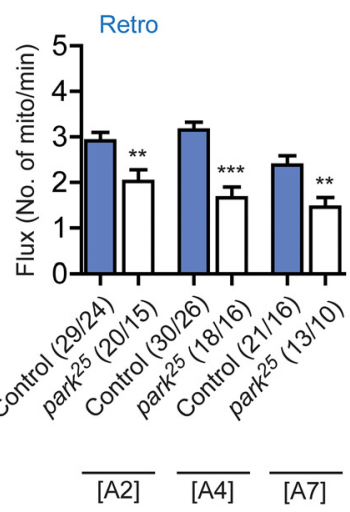

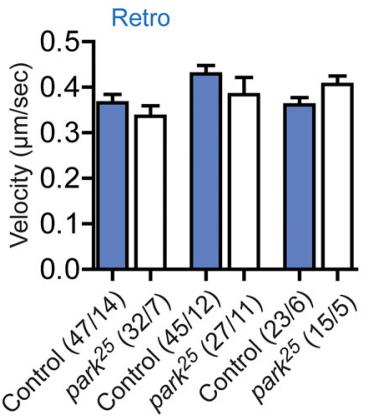

B
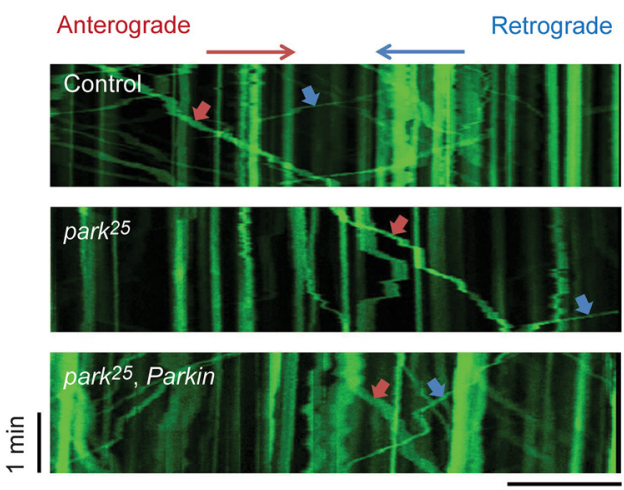

D

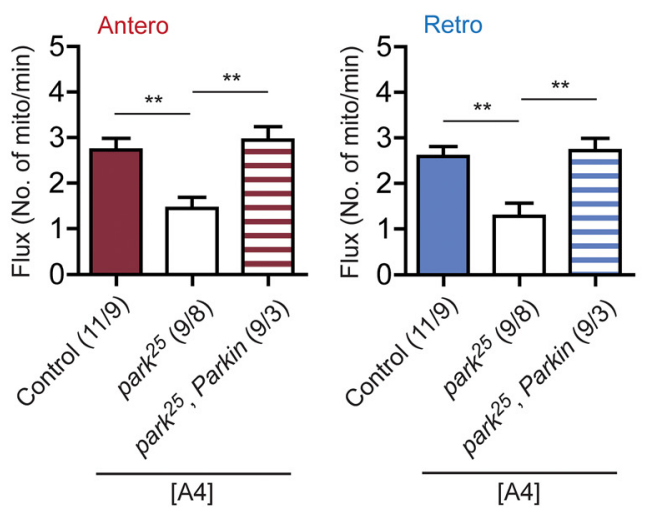

$\mathbf{F}$

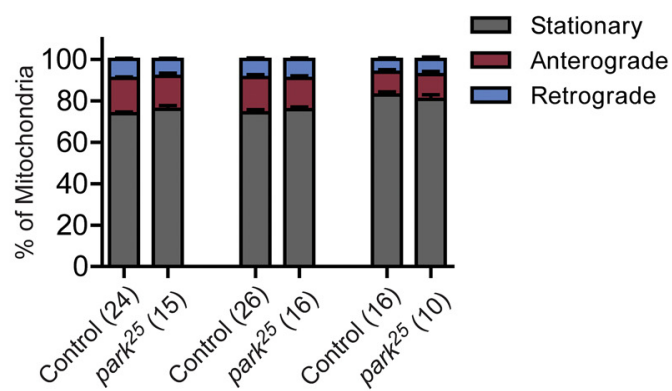

$\overline{[A 2]} \quad \overline{[A 4]} \quad \overline{[A 7]}$

Figure 1. Parkin perturbation alters mitochondrial axonal traffic by reducing flux without affecting motility properties. $A$, Confocal image of dissected late third instar larva expressing mito-GFP in motor neurons. Mitochondrial movements are monitored at three different regions in the axons of SNs: proximal (A2), middle (A4), and distal (A7). Thin branch nerves, adjacent NMJs, and cell bodies in the VG are also analyzed. Scale bar, $200 \mu \mathrm{m}$. B, Representative kymographs of mitochondrial axonal transport from the A4SNs. The red arrows indicate anterograde movements, while blue arrows indicate retrograde. Scale bar, $10 \mu \mathrm{m}$. C, Mitochondrial flux in different parkin genotypes in A2, A4, and A7 SNs. park $k^{25}$ mutants display attenuated mitochondrial flux in both directions throughout SNs. $(n / n)$, Number of axonal regions/number of animals. $D$, Mitochondrial flux in parkin deletion and rescue in A4 SNs. The attenuated mitochondrial flux in park ${ }^{25}$ mutants is restored by UAS-Parkin expression. $(n / n)$, Number of axonal regions/number of animals. $E$, Mitochondrial net velocity in different parkin genotypes. park $k^{25}$ mutants show retain normal mitochondrial velocities in both directions throughout the SNs. $(n / n)$, Number of mitochondria/number of animals. $\boldsymbol{F}$, The percentages of moving and stationary mitochondria are unaffected in park ${ }^{25}$ SNs. $n$, number of animals. Error bars indicate the mean \pm SEM. Significance is determined by Student's $t$ test $(\boldsymbol{C}, \boldsymbol{E}$, and $\boldsymbol{F})$ or by one-way ANOVA with Bonferroni correction $(\boldsymbol{D}) .{ }^{* *} p<0.01$ and ${ }^{* * *} p<0.001$.

will inhibit the turnover and/or retrograde traffic of mitochondria, causing an accumulation of senescent organelles in the axon. To test this hypothesis in vivo, we quantified the axonal transport of mitochondria in motor neurons of the intact Drosophila larval nervous system (Pilling et al., 2006; Russo et al., 2009). Using mito-GFP driven by $D 42$, transport was quantified in both directions in the proximal (segment A2), middle (segment A4), and distal (segment A7) regions of the SNs, and was compared between control (wild-type with D42-Gal4>UASmitoGFP) and park ${ }^{25}$ (parkin-null) animals (Fig. 1A,B; Devireddy et al., 2014). Consistent with previous analysis, mitochondrial flux in control motor axons was greater in the anterograde than in the 
Table 1. Duty cycle, run length, and area of axonal mitochondria from Drosophila larval motor neurons

\begin{tabular}{|c|c|c|c|c|c|c|}
\hline & \multicolumn{2}{|l|}{$\mathrm{A} 2$} & \multicolumn{2}{|l|}{ A4 } & \multicolumn{2}{|l|}{ A7 } \\
\hline & Anterograde & Retrograde & Anterograde & Retrograde & Anterograde & Retrograde \\
\hline \multicolumn{7}{|c|}{ Mitochondria duty cycle (\% of time) } \\
\hline \multicolumn{7}{|c|}{ Control } \\
\hline$S$ & $46.45 \pm 3.10$ & $48.17 \pm 2.94$ & $36.03 \pm 2.17$ & $35.90 \pm 2.16$ & $38.57 \pm 2.28$ & $45.37 \pm 3.97$ \\
\hline A & $51.83 \pm 3.01$ & $7.11 \pm 1.24$ & $60.46 \pm 2.39$ & $8.43 \pm 1.36$ & $60.26 \pm 2.57$ & $6.20 \pm 1.39$ \\
\hline $\mathrm{R}$ & $1.73 \pm 0.27$ & $44.73 \pm 2.50$ & $3.52 \pm 0.51$ & $55.67 \pm 2.53$ & $1.17 \pm 0.60$ & $48.43 \pm 5.01$ \\
\hline Mitochondria $(n)$ & 61 & 47 & 58 & 45 & 32 & 23 \\
\hline \multicolumn{7}{|l|}{ park $^{25}$} \\
\hline$S$ & $59.29 \pm 3.37^{*}$ & $59.54 \pm 3.50^{*}$ & $47.30 \pm 3.89^{*}$ & $53.88 \pm 5.80^{*}$ & $53.26 \pm 4.97^{*}$ & $51.71 \pm 6.61$ \\
\hline A & $39.83 \pm 3.42^{*}$ & $4.26 \pm 0.57$ & $50.20 \pm 3.88^{*}$ & $5.97 \pm 0.97$ & $45.44 \pm 5.10^{*}$ & $4.21 \pm 0.84$ \\
\hline $\mathrm{R}$ & $0.88 \pm 0.31$ & $36.20 \pm 3.29^{*}$ & $2.50 \pm 0.48$ & $40.16 \pm 5.39^{* *}$ & $1.30 \pm 0.47$ & $44.08 \pm 5.91$ \\
\hline Mitochondria $(n)$ & 35 & 32 & 48 & 27 & 15 & 15 \\
\hline \multicolumn{7}{|l|}{ Mitochondria run length ( $\mu \mathrm{m})$} \\
\hline Control & $2.44 \pm 0.13$ & $3.53 \pm 0.35$ & $3.08 \pm 0.21$ & $4.36 \pm 0.33$ & $2.96 \pm 0.45$ & $4.14 \pm 0.53$ \\
\hline Mitochondria (n) & 61 & 47 & 58 & 45 & 32 & 23 \\
\hline park $^{25}$ & $2.37 \pm 0.26$ & $3.58 \pm 0.44$ & $2.98 \pm 0.29$ & $3.51 \pm 0.20^{*}$ & $2.57 \pm 0.19$ & $3.73 \pm 0.31$ \\
\hline Mitochondria $(n)$ & 35 & 32 & 48 & 27 & 15 & 15 \\
\hline \multicolumn{7}{|l|}{ Mitochoncria area $\left(\mu \mathrm{m}^{2}\right)$} \\
\hline Control & $0.81 \pm 0.02$ & & $0.82 \pm 0.01$ & & $0.82 \pm 0.02$ & \\
\hline Mitochondria $(n)$ & 151 & & 204 & & 177 & \\
\hline park $^{25}$ & $0.80 \pm 0.01$ & & $0.79 \pm 0.03$ & & $0.77 \pm 0.02$ & \\
\hline Mitochondria (n) & 99 & & 69 & & 70 & \\
\hline
\end{tabular}

Quantitative analysis of axonal mitochondrial duty cycle, run length, and area from control and park ${ }^{25}$ animals at three different regions of SNs (A2, A4, and A7). To define duty cycle, moving mitochondria are considered as the percentage of time spent in a specific direction: $S$, stationary; $A$, anterograde; or $R$, retrograde. Run length is defined from moving mitochondria, while area is measured from axonal mitochondria regardless of movement. Data were acquired from at least three independent experiments and are expressed as the mean \pm SEM. $n$, Number of mitochondria. ${ }^{*} p<0.05$ and ${ }^{* *} p<0.01$.

retrograde direction, and declined with distance from the cell body in SNs (Shidara and Hollenbeck, 2010). In park ${ }^{25}$ SNs, flux was attenuated in both directions (Fig. 1C). However, anterograde flux was more severely impaired than retrograde in park ${ }^{25}$ SNs: anterograde flux dropped to $48 \%$ (A2), 41\% (A4), and 53\% (A7) of control, while retrograde flux dropped to $70 \%$ (A2), 53\% (A4), and 61\% (A7). These deficits were rescued by Parkin overexpression in the null background (Fig. $1 B, D$ ).

Because this pathway has been proposed to specifically detach kinesin from the mitochondrial surface (Wang et al., 2011), we next examined whether reduced mitochondrial flux in park $^{25} \mathrm{SNs}$ derived from the alteration of particular elements of mitochondrial motility. We analyzed the mitochondrial velocities, percentage moving, duty cycle, and run length (Devireddy et al., 2014). In park $^{25} \mathrm{SNs}$, mitochondrial velocities in both directions were similar to those of controls throughout the axons (Fig. 1E), and the percentages of moving and stationary mitochondria in motor axons were unaffected by Parkin deletion (Fig. $1 F$ ). This eliminated the major movement parameters as sources of the flux deficit, but we further measured the duty cycle and run length of moving mitochondria, and found that park ${ }^{25}$ mutants showed a modestly increased percentage of time pausing during mitochondrial movement in both directions, caused by the reduced proportion of movements in the dominant direction (Table 1). However, the run lengths of moving mitochondria were generally maintained with only a modest decrease in retrograde in the A4 region of SNs (dropped to $81 \%$; Table 1). These data indicate that although the loss of Parkin significantly reduces mitochondrial flux in the axon, mitochondrial motility behaviors and the percentage of moving mitochondria are nearly unchanged.

\section{Reduced mitochondrial flux in park $^{25}$ motor axons results mainly from reduced organelle density}

If mitochondrial motility was essentially normal in park ${ }^{25} \mathrm{mu}-$ tants, what could explain the large decrease in flux? One possibility is that Parkin deficiency reduces the number of mitochondria in the axon. This would run contrary to expectations, since the proposed role of the PINK1/Parkin pathway in mitochondrial turnover predicts that Parkin deficiency would cause the accumulation of senescent mitochondria (Narendra et al., 2008, 2010; Matsuda et al., 2010). Nonetheless, we assessed this possibility by quantifying total mitochondrial density (both moving and stationary) in SN axons (Devireddy et al., 2014) and found that there was indeed a pronounced loss of axonal mitochondria throughout park ${ }^{25}$ motor axons, as follows: the mitochondrial densities in axons were reduced to 52\% (A2), 61\% (A4), and 56\% (A7) of control levels in park ${ }^{25} \mathrm{SNs}$ (Fig. $2 A, B$ ). This deficit was restored in $\mathrm{A} 4$ of park $^{25} \mathrm{SNs}$ by Parkin overexpression (Fig. $2 A, C$ ). To examine this deficit in park $^{25}$ adult animals, we examined mitochondria in the wing, where a subset of sensory neurons expresses genes driven by $D 42$, and these are situated along the wing margin (L0 vein and L1 vein) with L3 vein (Fang et al., 2012). We visualized $D 42>$ mito-GFP mitochondria in these sensory axons by observing the HCV of LV1 (Fig. 2D). As in the larval nervous system, HCV axons of park ${ }^{25}$ mutant wings showed reduced mitochondrial density; unlike the larval SNs, the deficit was not significantly restored by Parkin overexpression (Fig. 2E).

Because the majority of axonal mitochondria in SNs are persistently stationary (Fig. $1 F$ ), we assessed separately the density of the moving mitochondria. We photo bleached the mito-GFP in a $30 \mu \mathrm{m}$ region of $\mathrm{SN}$, quantified the entry of mitochondria into it over time in both directions (Fig. $3 A$ ), and extrapolated the densities to steady state using both linear and hyperbolic plots with good agreement (Fig. $3 B, C$ ). The park ${ }^{25}$ axons showed a density of moving mitochondria $<60 \%$ that of controls, and this was partially rescued by Parkin overexpression. It was notable that, despite the lower densities of moving organelles, equal times were required to fill a bleached region to the steady state; both control and park $^{25}$ animals required $\sim 210$ s to reach a plateau (Fig. $3 B$ ). This confirms the previous observations (Fig. 1) that the reduced mitochondrial flux seen in park $^{25} \mathrm{SN}$ axons results from reduced organelle density without significant changes in motility behavior. 
A

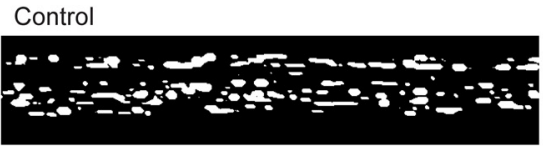

park $^{25}$

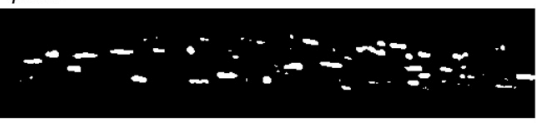

park $^{25}$, Parkin

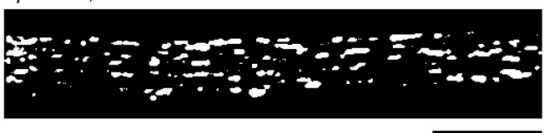

C

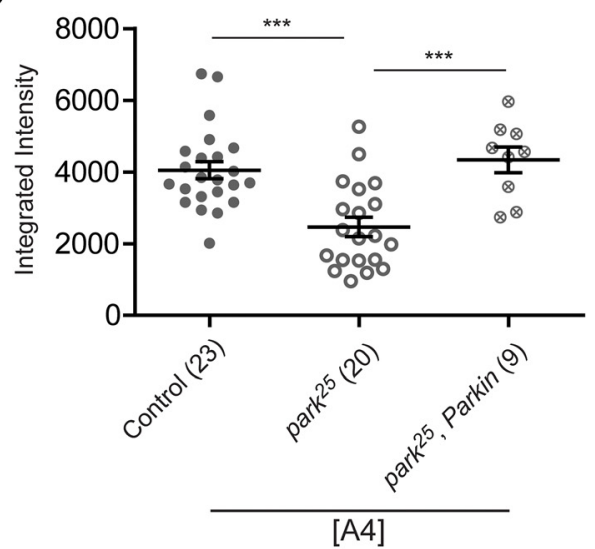

D

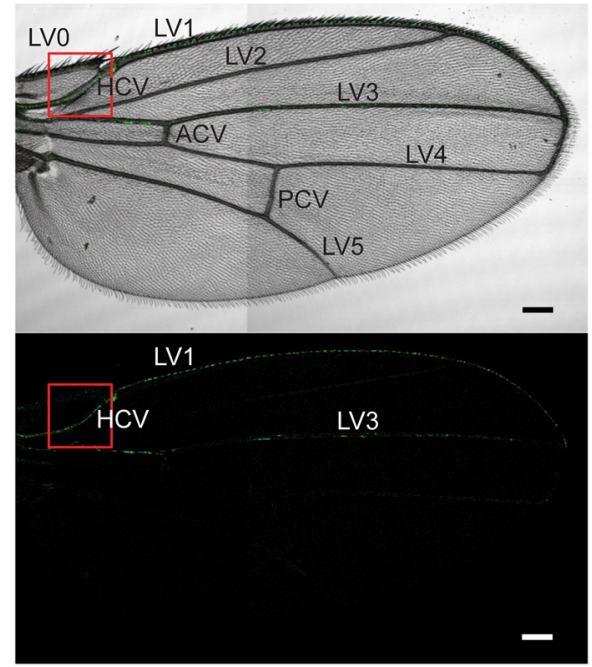

B

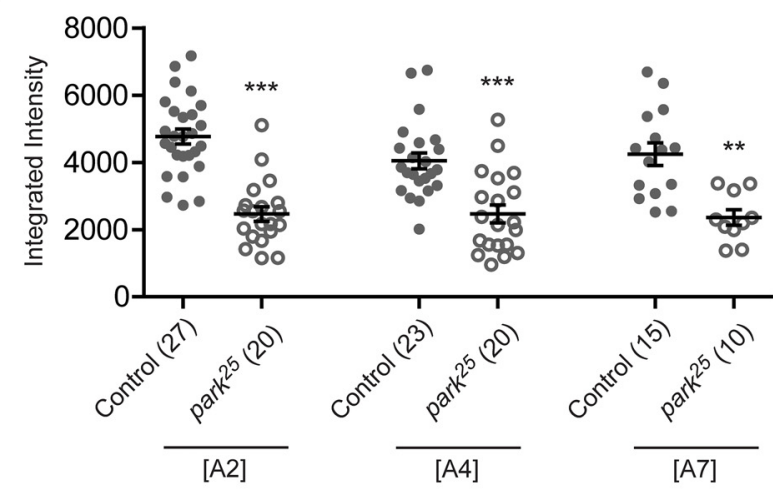

E
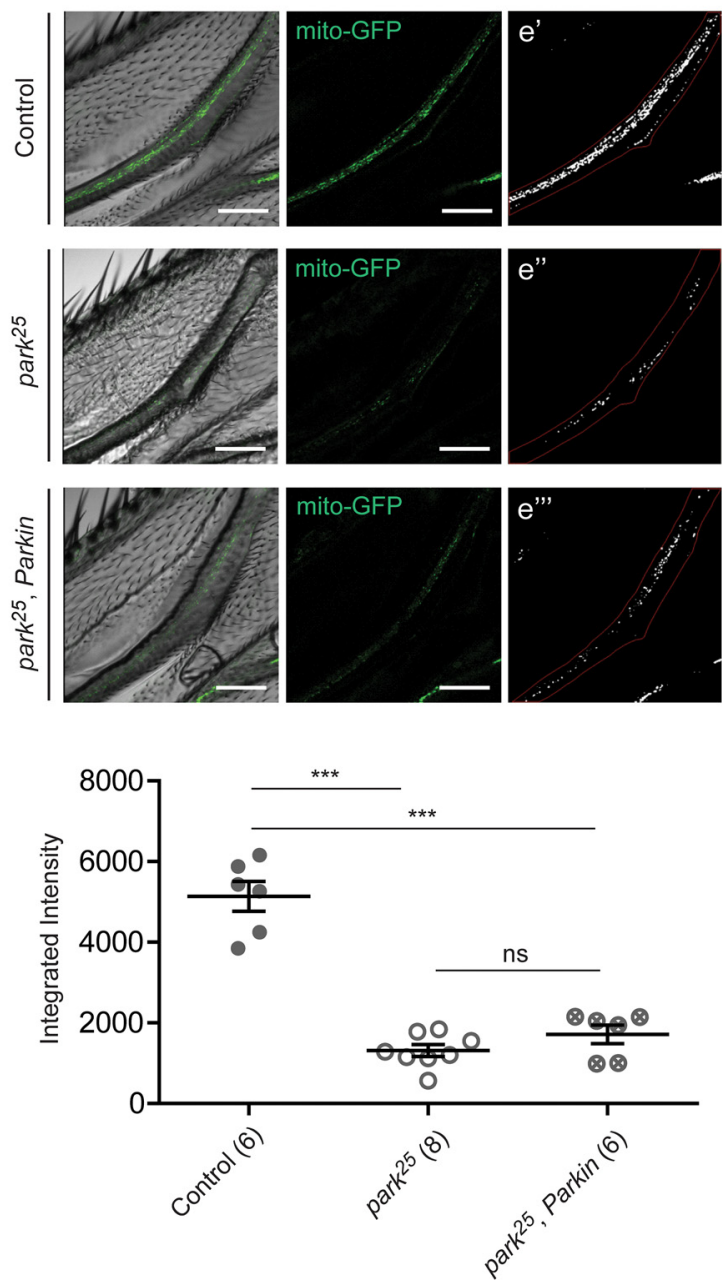

Figure 2. Reduced mitochondrial density is found in park ${ }^{25}$ motor axons. $\boldsymbol{A}$, Representative binarized images of mitochondria from the axon of A4 SNs. Scale bar, $10 \mu \mathrm{m}$. $\boldsymbol{B}$, Axonal mitochondrial density measurements from different parkin genotypes in the $A 2, A 4$, and $A 7$ regions show that density is decreased by half in park ${ }^{25}$ mutants throughout the axons. $C$, Axonal mitochondrial density from different parkin genotypes in A4 SNs shows that the decreased density in park ${ }^{25}$ axons is restored by UAS-Parkin expression. $D$, Axonal mitochondria from 5 -d-old adult fly wing. Axons from the HCV (red box) of the LV1 are monitored. Scale bars, $100 \mu \mathrm{m}$. $\boldsymbol{E}$, Axonal mitochondrial density in the HCV from different genotypes. Binarized images of mitochondria from the outlined area ( $\boldsymbol{e}^{\prime}, \boldsymbol{e}^{\prime \prime}$, and $\left.\boldsymbol{e}^{\prime \prime \prime}\right)$ are quantified, and park ${ }^{25}$ mutants display reduced mitochondrial density in HCV. The deficit is not restored by UAS-Parkin expression in adult HCV. Scale bars, $50 \mu \mathrm{m}$. $n$, number of animals. Error bars indicate the mean \pm SEM. Significance is determined by Student's $t$ test $(\boldsymbol{B})$ or by one-way ANOVA with Bonferroni correction $(\boldsymbol{C}, \boldsymbol{E})$. $^{* *} p<0.01$ and ${ }^{* * *} p<0.001$.

Parkin mutation affects neither gross morphology nor $\Delta \Psi_{m}$ of axonal mitochondria

The Parkin pathway has also been proposed to keep senescent, depolarized mitochondria from fusing with normal ones by promoting the degradation of Mitofusin (Deng et al., 2008; Poole et al., 2010; Ziviani et al., 2010). Thus, another prediction is that park $^{25}$ mutants will display longer mitochondria with diminished inner $\Delta \Psi_{\mathrm{m}}$. To test this hypothesis, we first quantified the length of both stationary and moving axonal mitochondria, and found that neither was affected by parkin perturbation (Fig. 4A). Stationary mitochondria measured $1.54 \pm 0.01 \mu \mathrm{m}$ in controls versus $1.55 \pm 0.01 \mu \mathrm{m}$ in park $^{25}$ mutants, while moving mito- 
A

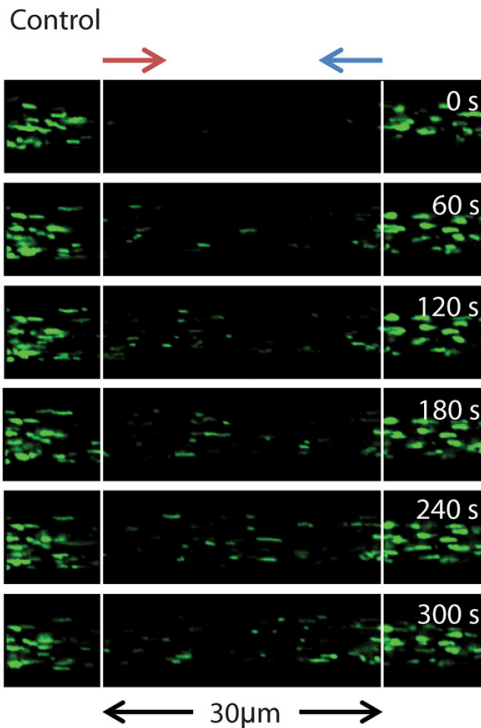

B

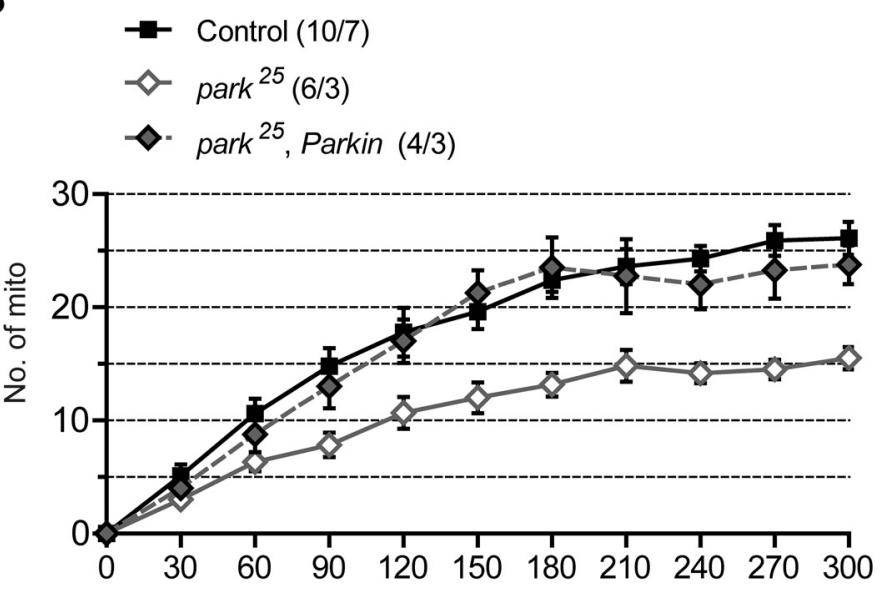

park $^{25}$
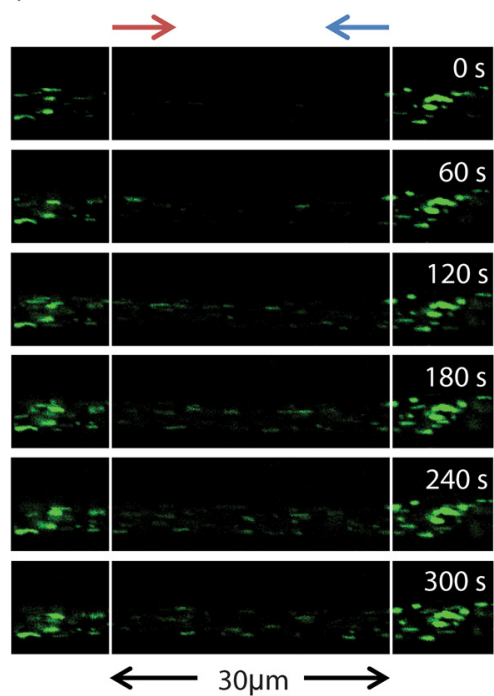

park $^{25}$, Parkin
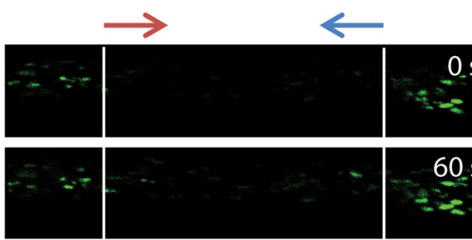

$60 \mathrm{~s}$

$t=$
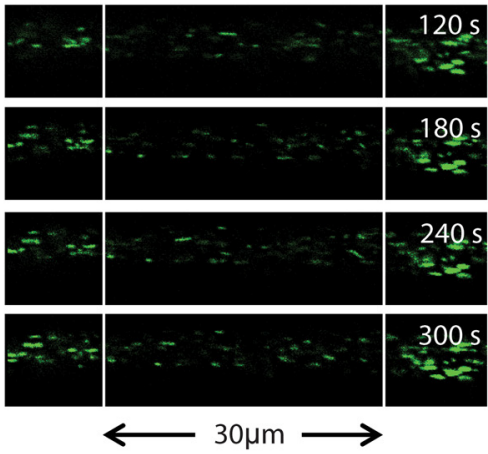

C
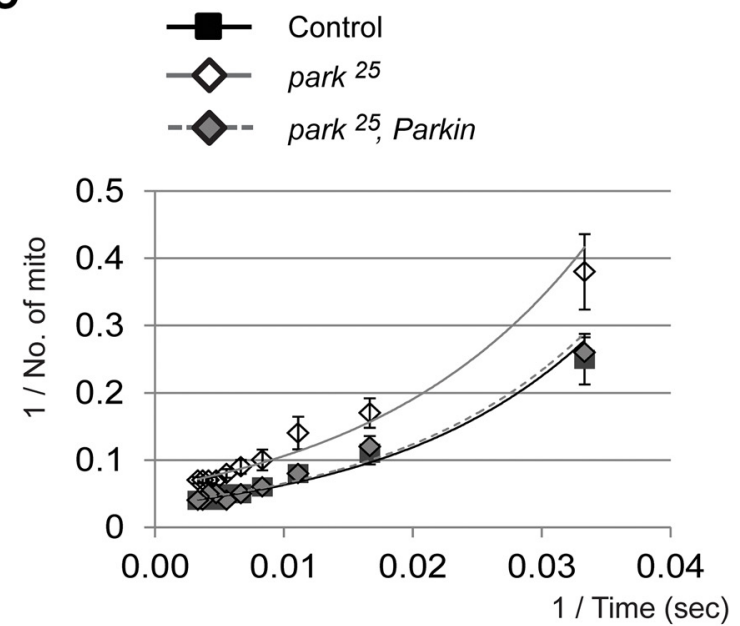

Figure 3. Reduced number of motile mitochondria is the cause of altered mitochondrial flux in park ${ }^{25}$ motor axons. A, Images from 5 min time lapse of a photo-bleached region of axon in A4 SNs. The steady-state numbers of moving mitochondria are quantified by monitoring their entry into the bleached regions $(30 \mu \mathrm{m})$. Red arrows indicate the anterograde direction, blue indicates retrograde. Scale bar, $10 \mu \mathrm{m} . \boldsymbol{B}$, Number of moving mitochondria in the bleached region reaches steady state at $\sim 5 \mathrm{~min}$. Mitochondria are counted every $30 \mathrm{~s}$. C, Double-reciprocal plots of the data in $\boldsymbol{B}$. The steady-state mitochondrial number is estimated from the extrapolated y intercepts. A total of 30 and 29 steady-state moving mitochondria are estimated in control and park ${ }^{25}$, Parkin animals, while 17 steady-state moving mitochondria are estimated in park $^{25}$ mutants from the $30 \mu \mathrm{m}$ photo-bleached regions. $(n / n)$, Number of axonal regions/number of animals. Error bars indicate the mean \pm SEM.

chondria were $1.38 \pm 0.03 \mu \mathrm{m}$ in controls versus $1.28 \pm 0.03 \mu \mathrm{m}$ in park ${ }^{25}$ mutants. To determine whether a length difference in park ${ }^{25}$ mutants might be manifested not in the population average, but as a small outlying population of longer mitochondria, we examined the length distributions by a two-sample Kolmogorov-Smirnov test and found no difference between controls and park $k^{25}$ mutants for either moving or stationary mitochondria (data not shown). We further measured the projected area of axonal mitochondria, to confirm whether park $k^{25}$ mutants show any shape differences in axonal mitochondria, and found that here too park $k^{25}$ measurements were comparable to those for controls throughout the axons (Table 1;0.82 $\pm 0.01 \mu \mathrm{m}^{2}$ with $0.56 \mu \mathrm{m}$ mean height in controls vs $0.79 \pm 0.01 \mu \mathrm{m}^{2}$ with 0.56 $\mu \mathrm{m}$ mean height in park ${ }^{25}$ mutants). Thus, Parkin seems to be dispensable for maintaining normal mitochondrial morphology in motor axons. We did not observe any correlation between morphology and motility in SN axons, so we further regressed mitochondrial velocities against their lengths to divine any relationship between the two. Although lengths were distributed from 0.35 to $4.65 \mu \mathrm{m}$, the net velocities of mitochondria were consistent in both directions, and there was no correlation between length and velocity or direction of movement (data not shown). Although this information seems counterintuitive on its face, it is consistent with the findings of previous studies that have shown no effect of mitochondrial length on axonal motility except at extreme, nonphysiological lengths (Amiri and Hollenbeck, 2008).

Next, we assessed the $\Delta \Psi_{\mathrm{m}}$ of mitochondria from intact larval motor neurons to verify whether the sparse axonal mitochondria in park $^{25}$ mutants nonetheless display the diminished membrane potential that is characteristic of senescence. TMRM was used for quantitative determination of transmembrane potential using the $\mathrm{F}_{\mathrm{m}} / \mathrm{F}_{\mathrm{c}}$ ratio in mitochondria in thin peripheral motor nerves and NMJs adjacent to the larval A4 segment in vivo (Verburg and 
A

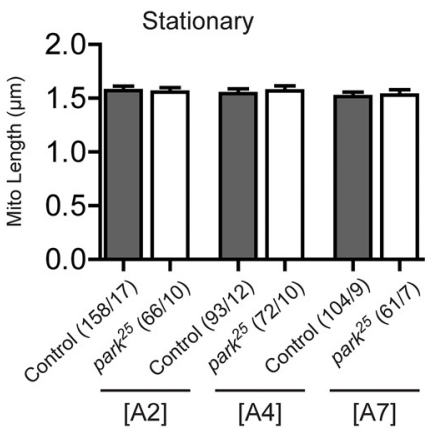

B
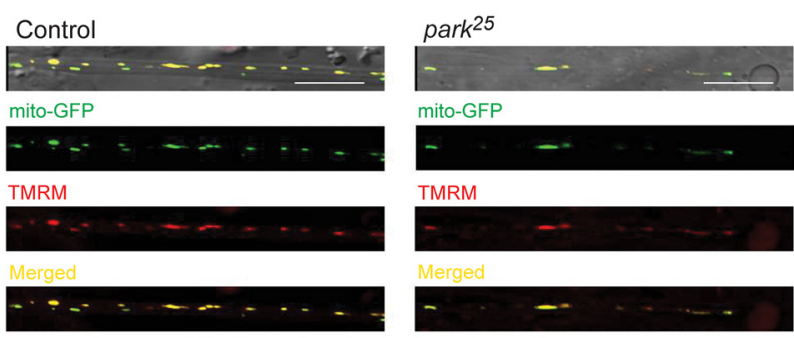

D
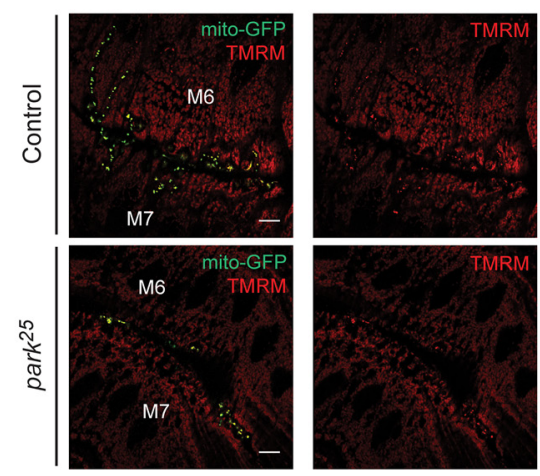

F
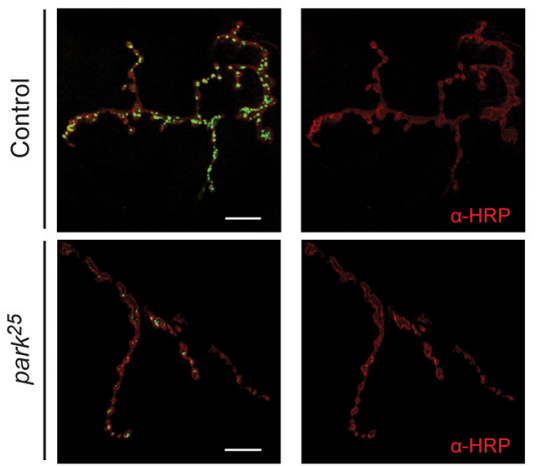
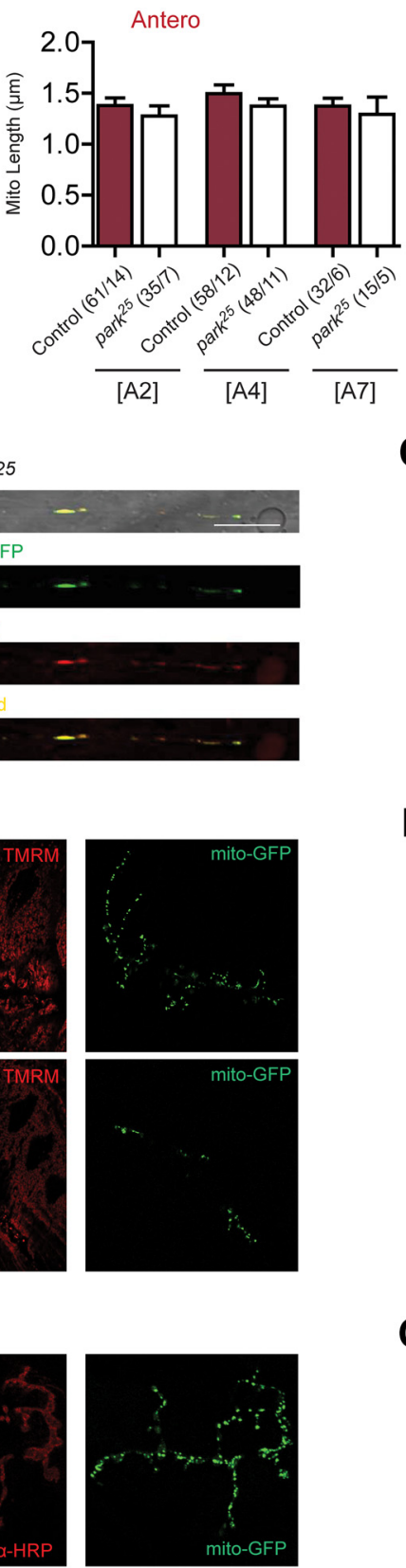

C

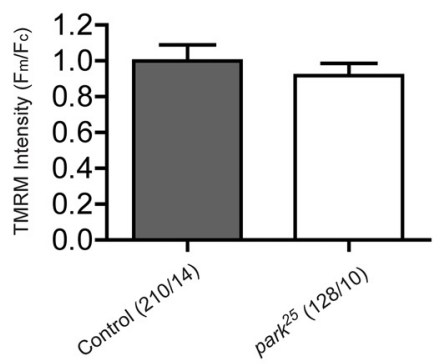

E

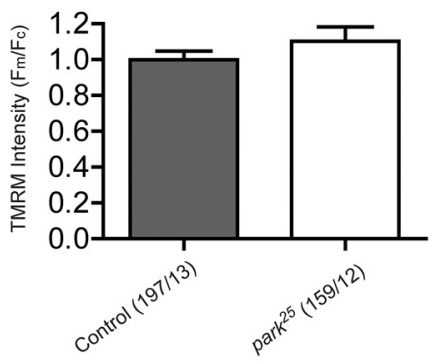

G

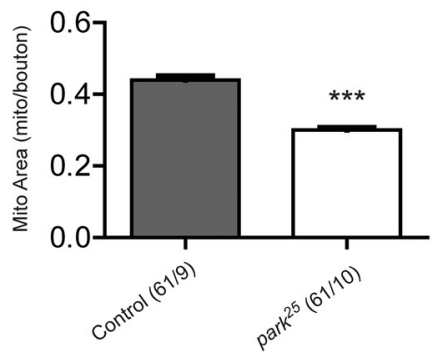

Figure 4. Parkin-deficient animals do not accumulate senescent mitochondria in motor axons and NMJs. $\boldsymbol{A}$, Mean length of axonal mitochondria in different parkin genotypes. Stationary and moving mitochondria are measured in axons of A2, A4, and A7 SNs. Normal mitochondrial length is preserved in park ${ }^{25}$ mutants throughout the SNs. $\boldsymbol{B}$, Representative TMRM stained images of axonal mitochondria with mito-GFP. Mitochondria are stained with TMRM (red) to indicate inner $\Delta \Psi_{\mathrm{m}}$, while mito-GFP (green) reveals all axonal mitochondria. Note that mito-GFP here confirms the lower mitochondrial density in park ${ }^{25}$ axons compared with controls. Scale bars, $10 \mu \mathrm{m}$. $\boldsymbol{C}$, Mean $\mathrm{F}_{\mathrm{m}} / \mathrm{F}_{\mathrm{c}}$ intensity ratio of TMRM from neurons in different parkin genotypes. Normal mitochondrial inner membrane potential is retained in park ${ }^{25}$ mutant axons. (n/n), Number of mitochondria/number of animals. $\boldsymbol{D}$, Representative TMRM staining images of mitochondria with mito-GFP from NMJs. NMJs are taken between muscles 7 (M7) and 6 (M6) from A4 SNs, and mitochondria in synaptic boutons are selected for $\Delta \Psi_{\mathrm{m}}$ quantification. Scale bars, $10 \mu \mathrm{m}$. $\boldsymbol{E}$, Mean $\mathrm{F}_{\mathrm{m}} / \mathrm{F}_{\mathrm{c}}$ intensity ratio of TMRM from NMJs in different genetic backgrounds. Normal $\Delta \Psi_{\mathrm{m}}$ is retained in synaptic boutons of park ${ }^{25}$ NMJs. $(n / n)$, Number of mitochondria/number of animals. $\boldsymbol{F}$, Images of mito-GFP (green) and anti-HRP staining (red) show mitochondria in motor synaptic boutons. Scale bars, $10 \mu$ m. $\mathbf{G}$, Mitochondrial density in motor NMJs. park ${ }^{25}$ mutants display reduced mitochondrial density in synaptic boutons. ( $\left.n / n\right)$, Number of boutons/number of animals. Error bars indicate the mean \pm SEM. Significance is determined by Student's $t$ test. ${ }^{* * *} p<0.001$. 
A
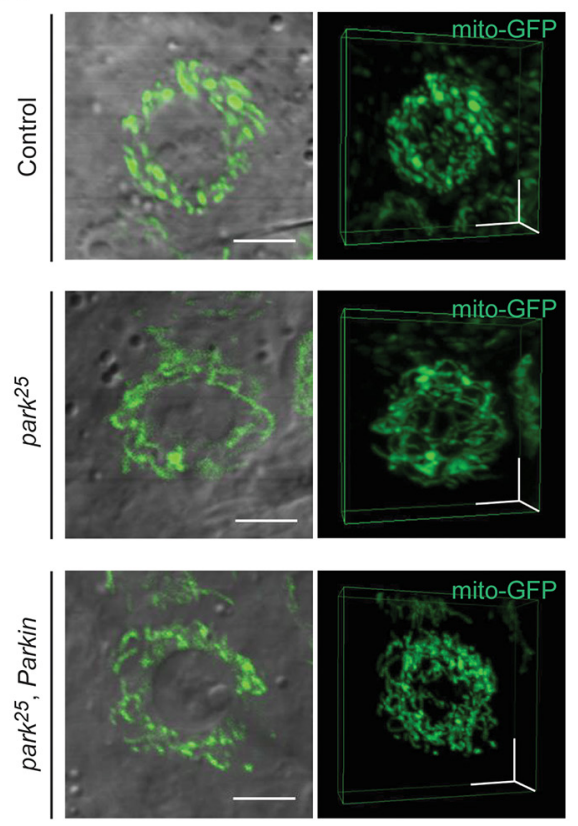

B
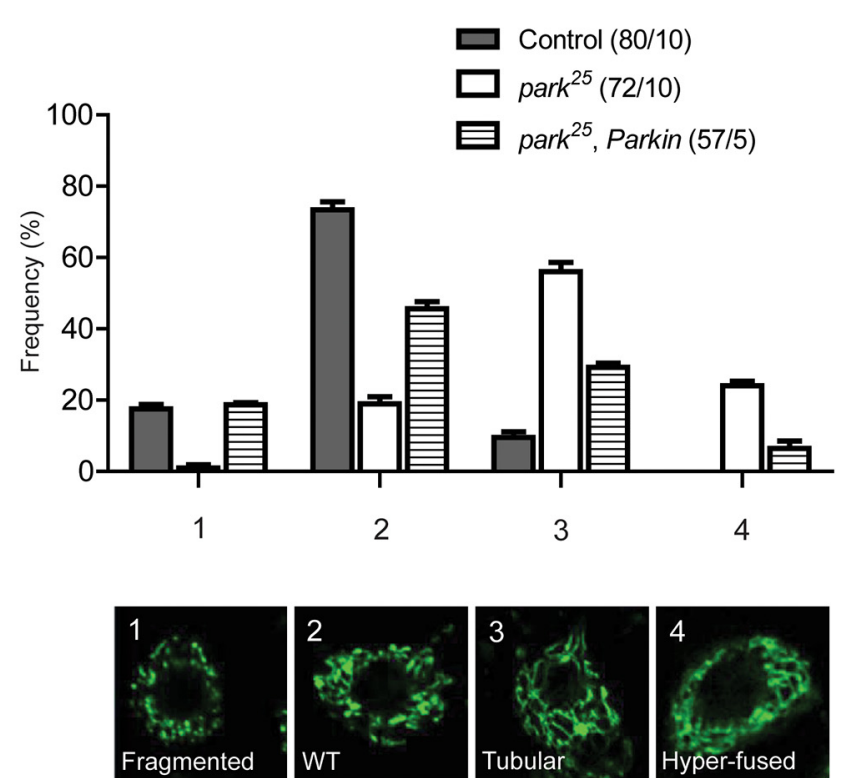

Figure 5. Parkin perturbation produces tubular, interconnected mitochondria in cell bodies. $A$, Representative plane and three-dimensional reconstructed images of the mitochondria in motor cell bodies. Reconstructed images are generated from sections of $20 \mu \mathrm{m}$ width $(x), 20 \mu \mathrm{m}$ height $(y)$, and $5 \mu \mathrm{m}$ depth ( $z)$ using 100 frames to construct the $z$-stacks. Scale bars, $5 \mu \mathrm{m}$. $\boldsymbol{B}$, Mitochondrial morphology in cell bodies of different parkin genotypes. Mitochondrial morphology is categorized as follows: 1, fragmented; 2 , wild-type; 3 , tubular; or 4, hyperfused. Tubular and hyperfused morphological defects of mitochondria are observed much more frequently in park ${ }^{25}$ cell bodies. Morphological defects of mitochondria in park ${ }^{25}$ cell bodies are partially restored by UAS-Parkin expression. $(n / n)$, Number of cell bodies/number of animals. Data are generated in three independent analyses blinded to the genotype in the images and assessed by a two-sample Kolmogorov-Smirnov test (morphological changes of mitochondria caused by Parkin-null are significantly different from control; $D=0.7306>$ critical $D=0.2209$ with a corresponding $p<$ $0.001)$.

Hollenbeck, 2008; Shidara and Hollenbeck, 2010; Devireddy et al., 2014, 2015). Surprisingly, the $\Delta \Psi_{\mathrm{m}}$ values in park ${ }^{25} \mathrm{SNs}$ (Fig. $4 B, C$ ) and NMJs (Fig. $4 D, E$ ) was indistinguishable from those in controls, and the reduced mitochondrial density observed in SN axons was also apparent in these thin nerves (Fig. 4B) and synaptic boutons (Fig. 4F, G) of park ${ }^{25}$ mutants. Our results thus indicate that Parkin deletion fails to produce the predicted accumulation of longer and senescent mitochondria in motor axons in vivo; park $^{25}$ mutant animals display normal status for their axonal mitochondria.

\section{Parkin deficit produces abnormal mitochondrial morphology} in motor cell bodies

Parkin deficiency not only failed to produce the changes in axons predicted by its proposed role in mitochondrial QC-that is, an accumulation of senescent, longer mitochondria-but actually generated a dramatically reduced density of axonal mitochondria. This raised the possibility that the cell body is the locus of Parkin-dependent turnover and/or Parkin-dependent mitochondrial biogenesis, perhaps including the regulation of axonal entry. Thus, we analyzed the morphology of mitochondria in motor cell bodies of the larval nervous system; these give rise to the SN motor axons observed above. Interestingly, abnormal tubular, interconnected mitochondria were found in park $^{25}$ cell bodies (Fig. 5A). To quantify these complex differences in organelle morphology, we categorized the cell body mitochondria into the following four different phenotypes-fragmented (1), wildtype (2), tubular (3), and hyperfused (4) - and scored them while blinded to the genotype. Compared with controls, the park ${ }^{25}$ cell bodies showed a much higher frequency of tubular (56\% vs 9.6\%) and hyperfused (24\% vs $0 \%$ ) mitochondria (Fig. $5 B$ ). Overexpression of Parkin in the park ${ }^{25}$ mutant background largely restored normal mitochondrial morphology (Fig. 5A,B). Together with Figure 3, these data indicate that Parkin is essential for both normal mitochondrial morphology in the cell body and the entry of mitochondria into the axon, and suggest the following likely relation of the two: that normal somatic organelle morphology, reflecting a normal fission-fusion balance, is important for producing and/or launching axonal mitochondria.

\section{Parkin-dependent mitophagy is undetectable in motor neurons in vivo}

Studies in embryonic neurons in vitro have shown that autophagosomes initiate distally and undergo maturation during their retrograde transport (Maday et al., 2012; Maday and Holzbaur, 2014). Since the PINK1/Parkin pathway is thought to be necessary for local mitophagy in the distal axon (Ashrafi et al., 2014), the lack of evidence for Parkin-dependent axonal mitochondrial turnover that we found here was surprising. To probe further the role of Parkin in mitophagy in vivo, we visualized simultaneously mitochondria and AVs in SN motor neurons, using D42-driven mito-GFP and RFP-Atg8 (Barth et al., 2011). Because we had observed altered mitochondrial morphology in park $^{25}$ cell bodies, we first quantified the colocalization of AVs with mitochondria there to determine whether Parkin plays a critical role in targeting dysfunctional mitochondria for mitophagy. Consistent with our previous data, tubular and elongated mitochondria were found in park $^{25}$ cell bodies. However, mitochondria failed almost completely to colocalize with AVs in either control or park ${ }^{25}$ cell bodies (Fig. 6A,B). Studies using in vivo imaging of autophagosomes in mouse Purkinje cells in the cerebellar cortex provide evidence that macroautophagy is induced by starvation (Alirezaei et al., 2010; Chen et al., 2015). Thus, to increase autophagy above basal conditions, we prevented larvae from feeding for $6 \mathrm{~h}$ before ob- 
A
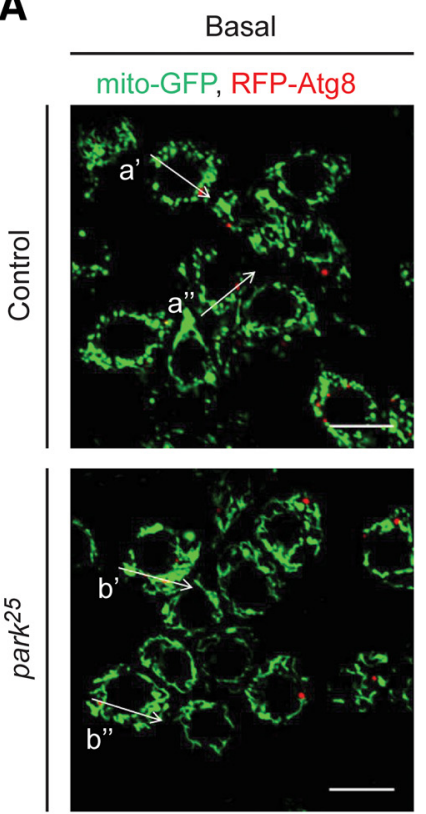

Starvation (6h)
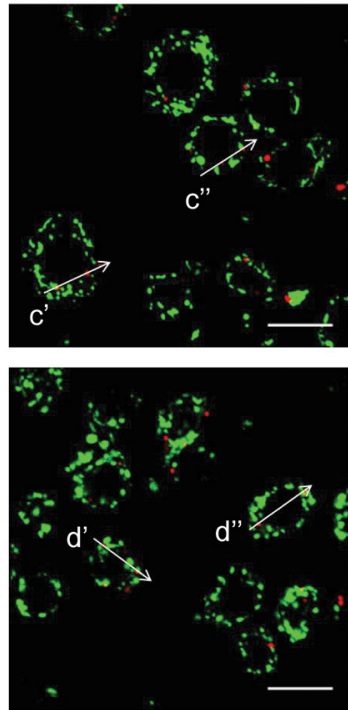

B
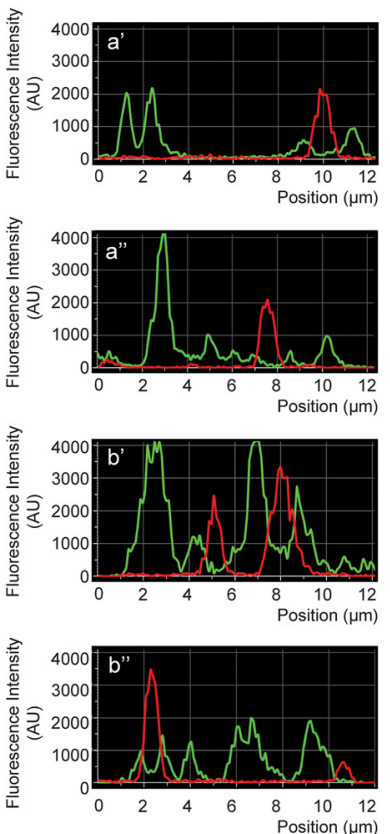
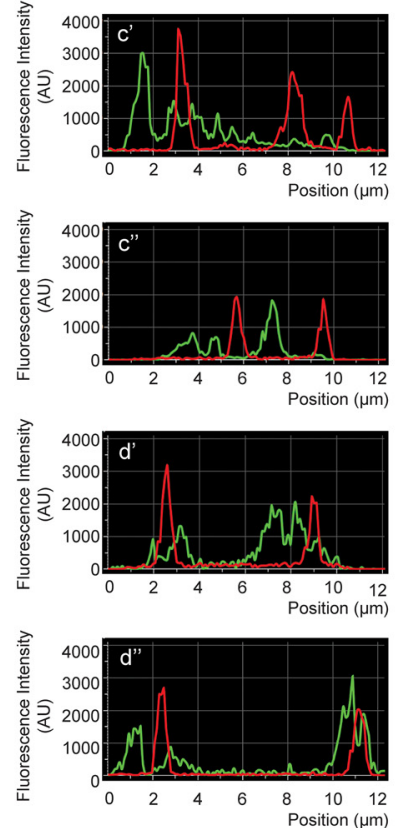

C

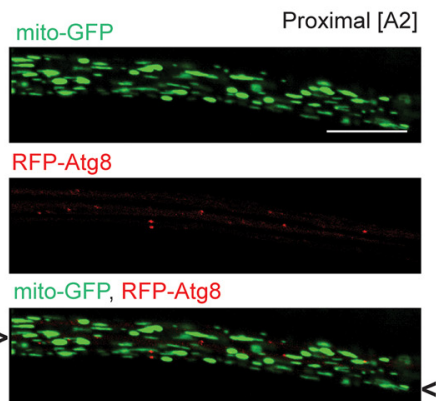

D
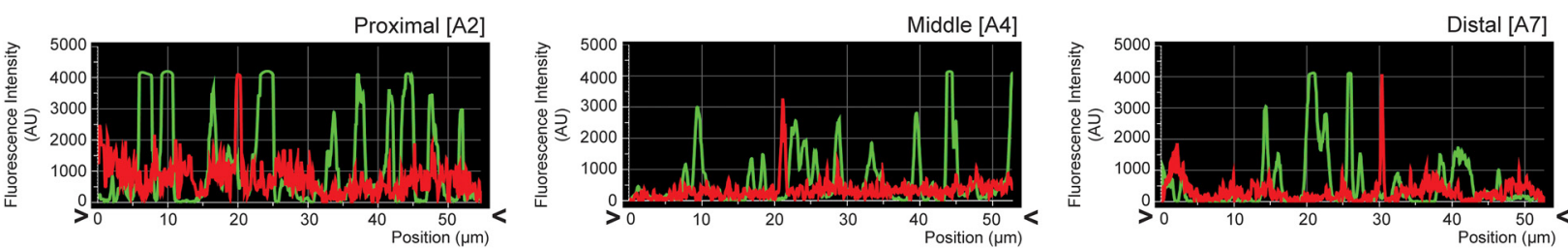

E

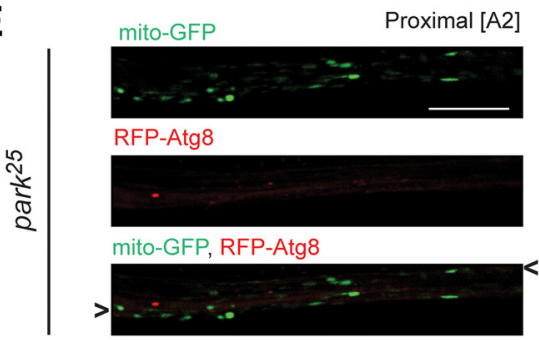

F

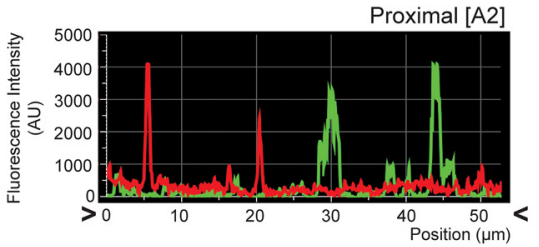

mito-GFP

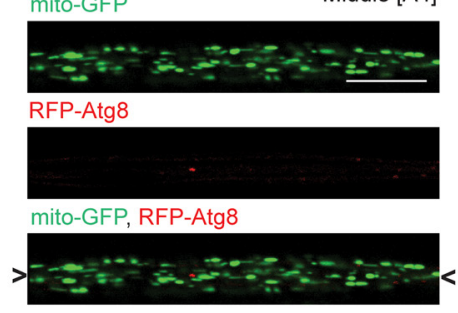

mito-GFP

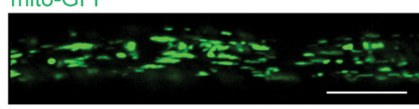

RFP-Atg8

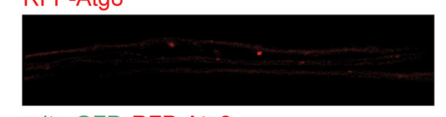

mito-GFP, RFP-Atg8

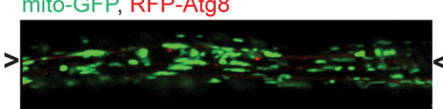

mito-GFP

Middle [A4]

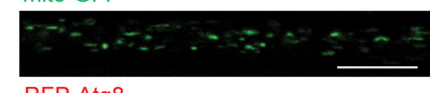

RFP-Atg8

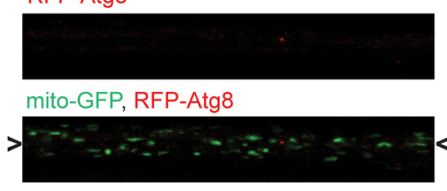

Middle [A4]

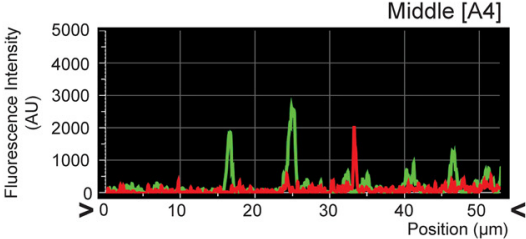

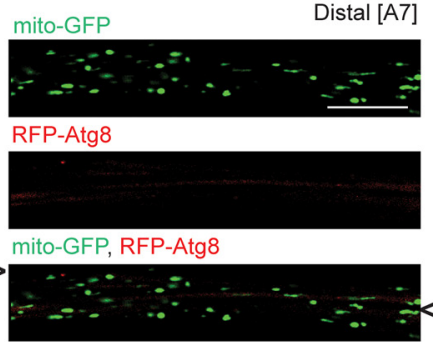

Distal [A7]

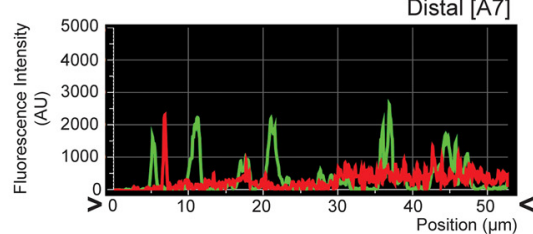

Figure 6. Mitochondria do not colocalize significantly with AVs in vivo. $\boldsymbol{A}$, Representative images of cell bodies in different parkin genotypes. mito-GFP fluorescence is used to identify mitochondria (green) and RFP-Atg8 to identify AVs (red). Autophagy is induced by $6 \mathrm{~h}$ of starvation. White arrows indicate lines along which organelle colocalization is analyzed for basal ( $\boldsymbol{a}^{\prime}, \boldsymbol{a}^{\prime \prime}, \boldsymbol{b}^{\prime}$, and $\left.\boldsymbol{b}^{\prime \prime}\right)$ and starvation $\left(\boldsymbol{c}^{\prime}, \boldsymbol{c}^{\prime \prime}, \boldsymbol{d}^{\prime}\right.$, and $\left.\boldsymbol{d}^{\prime \prime}\right)$ conditions. Scale bars, $10 \mu \mathrm{m}$. $\boldsymbol{B}$, Respective signal intensity profiles from the line scans indicate in $\boldsymbol{A}$. Distinct mito-GFP and RFP-Atg8 signals reveal no clearly detectable mitophagy events in motor cell bodies from either genotype. $\boldsymbol{C}$, Representative images of axonal mitochondria with AVs in A2, A4, and A7 SNs from control animals. Organelle colocalization is represented by oblique (A2) or rectilinear (A4 and A7) line scan analysis. Scale bars, $10 \mu \mathrm{m}$. D, Signal intensity profiles of line scans of images in C. (Figure legend continues.) 
A
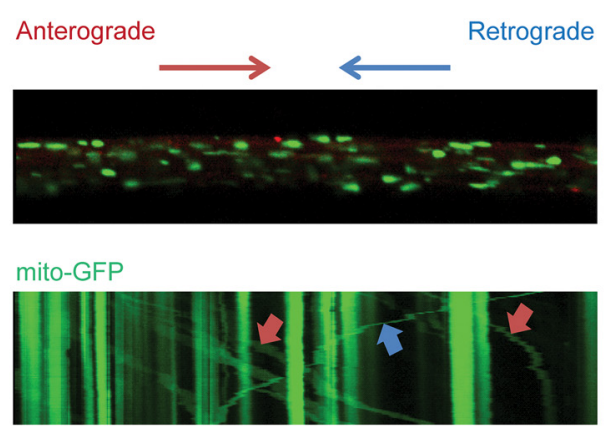

RFP-Atg8
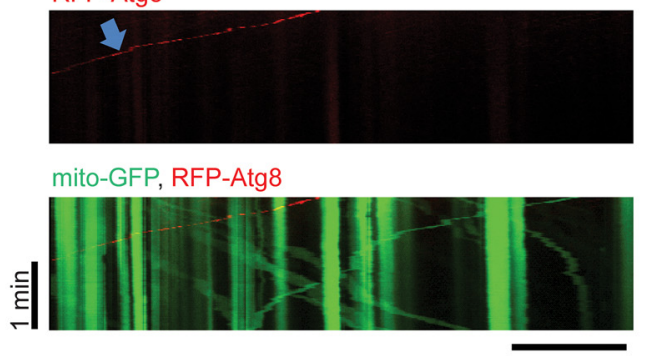

B
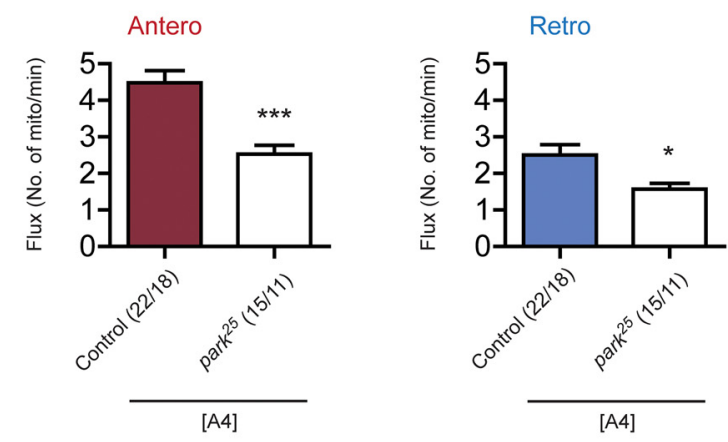

C

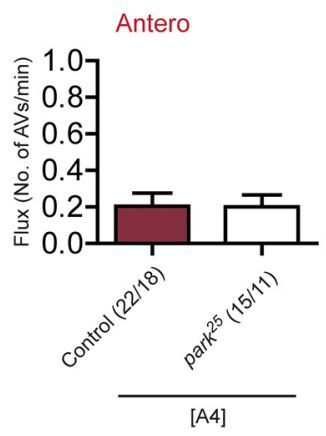

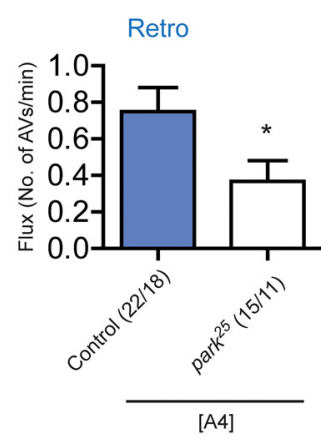

Figure 7. Parkin perturbation impairs retrograde transport of AVs. A, Representative kymographs of mitochondrial and autophagosomal axonal transport from the A4 SNs axons of control animals. Red arrows indicate anterograde moving mitochondria, and blue arrows indicate retrograde moving organelles. Scale bar, $10 \mu \mathrm{m}$. $\boldsymbol{B}$, Mitochondrial flux in different parkin genotypes. C, Autophagosomal flux in different parkin genotypes. Reduced AV flux is observed only in the retrograde direction, while reduced mitochondrial flux is observed in both directions in park ${ }^{25}$ mutant axons. $(n / n)$, Number of axonal regions/number of animals. Error bars indicate the mean \pm SEM. Significance is determined by Student's $t$ test. ${ }^{*} p<0.05$ and ${ }^{* * *} p<0.001$.

servation, triggering starvation-induced autophagy (Gomes et al., 2011; Rambold et al., 2011; Ghosh et al., 2012). Although this treatment caused mitochondrial fragmentation (Fig. 6A), which should render mitochondria even better candidates for engulfment, it did not generate detectable colocalization of AVs with mitochondria in either control or park ${ }^{25}$ cell bodies (Fig. 6A,B). The mito-GFP and RFP-Atg8 signals clearly represented distinct organelles in virtually every case. Thus, in cell bodies we could not test the hypothesis that Parkin deficiency would reduce the rate of mitophagy in vivo, because there was no detectable mitophagy even in controls, and under either normal physiological or starvation-induced autophagic conditions.

We then observed autophagosomes along motor axons to elucidate the organization of autophagy and mitophagy in that compartment. As expected from previous studies (Maday et al., 2012), axonal AVs mainly moved retrogradely and rarely changed their direction, yielding a retrograde/anterograde flux ratio of almost 4, quite distinct from the saltatory bidirectional movements of mitochondria (Fig. 7A). The majority of retrograde AVs displayed faster movement than mitochondria (data not shown). When we simultaneously analyzed axonal transport of mitochondria and AVs, we found that, consistent with our

\section{$\leftarrow$}

(Figure legend continued.) $\boldsymbol{E}$, Representative images of axonal mitochondria with $A V s$ in $A 2, A 4$, and A7 SNs from park ${ }^{25}$ mutant animals. Organelle colocalization is determined by oblique (A2 and A7) or rectilinear (A4) line scan analysis. Scale bars, $10 \mu \mathrm{m}$. F, Signal intensity profiles of line scans of the images in $E$. Mito-GFP and RFP-Atg8 signals from both genotypes show distinct, nonoverlapping distributions of mitochondria and AV signal throughout SNs. Angle brackets denote corresponding points in images $(\boldsymbol{C}, \boldsymbol{E})$ and line scan analysis for intensity profiles $(\boldsymbol{D}, \boldsymbol{F})$. AU, Arbitrary units. previous data, mitochondrial flux was diminished in both directions by Parkin deficiency (Fig. 7B). AV flux was also substantially reduced in park ${ }^{25}$ mutants, but specifically in the retrograde direction; park $^{25}$ mutants showed anterograde AV flux equal to that of controls, while retrograde flux was reduced by $50 \%$ (Fig. $7 C)$. However, as in cell bodies, in axons we observed a striking lack of colocalization of AVs with mitochondria in either control or park ${ }^{25}$ mutant animals (Fig. $6 C-F$ ). These results indicate that while AVs are obvious and their axonal transport is consistent with them having a Parkin-dependent origin and/or maturation in the distal axon, mitophagy is so rare as to be undetectable in motor neurons in vivo. This was true even when starvation (Fig. $6 A, B$ ) produced large numbers of small, fragmented mitochondria that are highly suitable for autophagic engulfment.

\section{Mitophagy is common in Drosophila neurons in vitro and requires Parkin}

Although we did not observe mitochondrial colocalization with AVs in Drosophila neurons in vivo, we note that most of the previous studies that have supported a role for Parkin in mitochondrial turnover and mitophagy were performed in vitro, with either neuronal cells (Ashrafi et al., 2014) or non-neuronal cells (Narendra et al., 2008, 2010; Matsuda et al., 2010; Lazarou et al., 2015). Thus, we examined whether autophagosomes and mitophagy can be detected in neurons in vitro in the same system that we used for our in vivo studies. We grew Drosophila primary motor neurons in culture, using the mito-GFP larvae as our source of cells (Bai et al., 2009; Pathak et al., 2010). In contrast to our in vivo results, we observed numerous AVs that colocalized with mitochondrial signals in vitro (Fig. $8 A$ ). Not only did control 
A
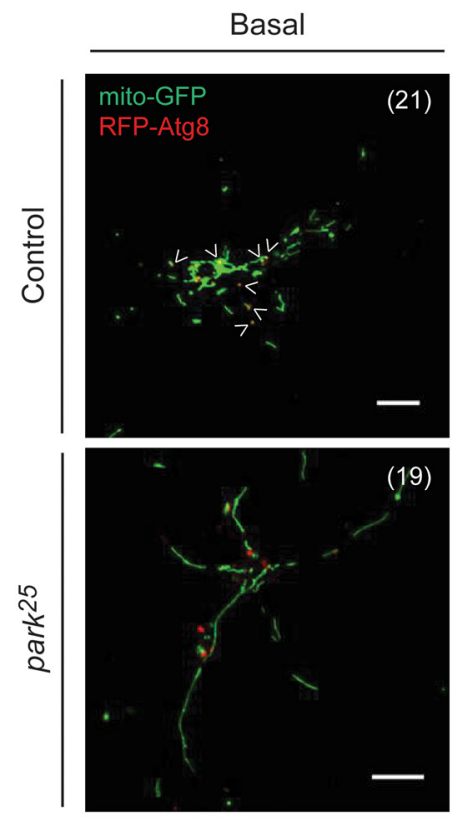

B
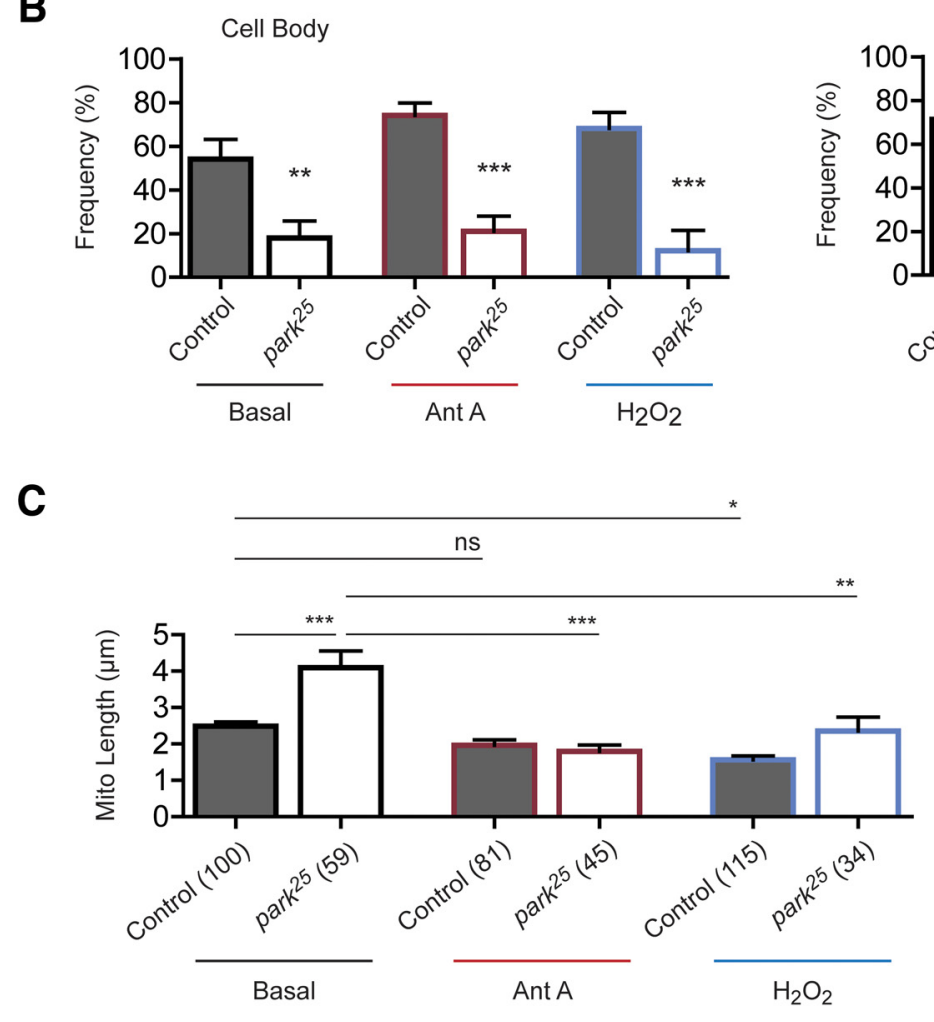

C
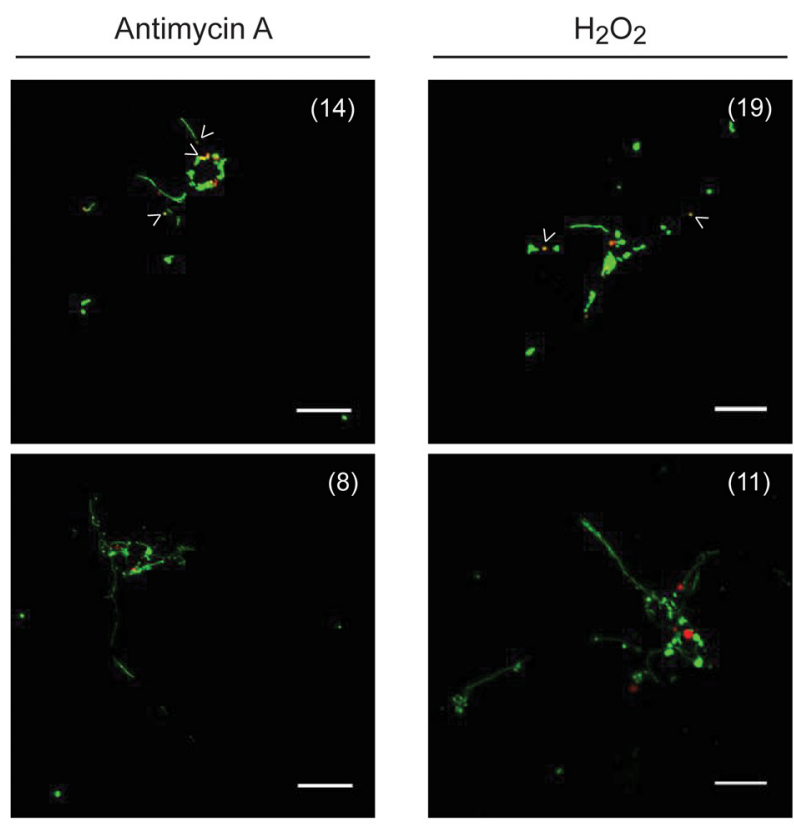

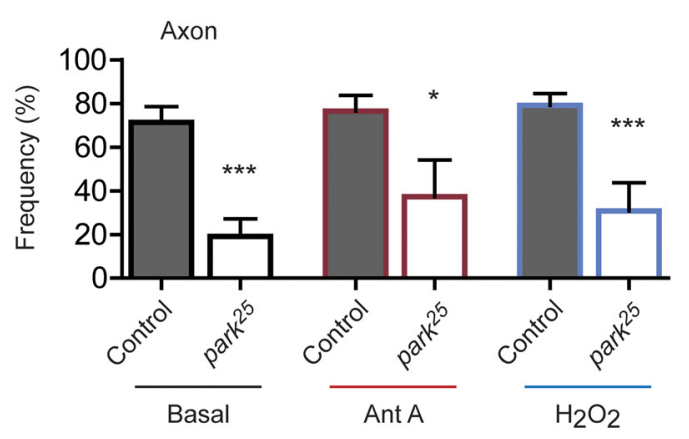

Figure 8. Parkin is essential for mitochondrial engulfment by autophagosomes in vitro. A, Images of in vitro primary motor neurons expressing mito-GFP and RFP-Atg8 after $96 \mathrm{~h}$ in culture. For stress conditions, cells are exposed to Ant A ( $5 \mu \mathrm{m}, 20 \mathrm{~min})$ or $\mathrm{H}_{2} \mathrm{O}_{2}(100 \mu \mathrm{M}, 1 \mathrm{~h})$ before imaging. White angle brackets denote the colocalization of mitochondria with AVs. Scale bars, $10 \mu \mathrm{m}$. $n$, Number of cells. B, Frequency of autophagosomes that contain mitochondria under each condition. Mitochondrial engulfment into AVs is inhibited in park ${ }^{25}$ mutant neurons. $\boldsymbol{C}$, Mean length of axonal mitochondria in different parkin genotypes in exposed conditions. park ${ }^{25}$ mutant neurons show elongated axonal mitochondria. Axonal mitochondrial elongation in park ${ }^{25}$ neurons is inhibited by either treatment ( $\mathrm{Ant} \mathrm{A} \mathrm{or} \mathrm{H}_{2} \mathrm{O}_{2}$ ). $n$, Number of mitochondria. Error bars indicate the mean \pm SEM. Significance is determined by Student's $t$ test $(\boldsymbol{B})$ or by one-way ANOVA with Bonferroni correction (C). ${ }^{*} p<0.05,{ }^{* *} p<0.01$, and ${ }^{* * *} p<0.001$.

neurons show this evidence for mitophagy in vitro, but the frequency of AV-mitochondrial colocalization was substantially reduced in neurons from park ${ }^{25}$ mutant larvae; frequencies were reduced to $33 \%$ of control levels in the cell bodies and $27 \%$ in the axons (Fig. $8 B$ ). This suggests that mitochondrial engulfment into autophagosomes is Parkin-dependent in vitro. We also ob- served more elongated axonal mitochondria in park ${ }^{25}$ mutant neurons in vitro (Fig. $8 \mathrm{~A}, \mathrm{C}$ ), which is predicted by current models of the role of Parkin in mitochondrial turnover (Deng et al., 2008), but was not observed in vivo. However, the number of AVs per cell in neurons cultured from park ${ }^{25}$ mutants was not reduced relative to controls in either the cell bodies or the axons (data not 
shown), arguing against Parkin control of general autophagy in vitro.

In neuronal (Ashrafi et al., 2014) as well as non-neuronal (Matsuda et al., 2010; Narendra et al., 2010) environments, mitochondria that undergo mitophagy mostly show an isolated, fragmented morphology. Thus, we further asked whether the size of mitochondria alone affects mitophagy, as visualized by AVmitochondrial colocalization. To remodel mitochondria independently of Parkin perturbation, neurons were treated with $5 \mu \mathrm{M}$ Ant A, an inhibitor of respiratory complex III that depolarizes neuronal mitochondria (Slater, 1973; Cai et al., 2012). Applying $5 \mu \mathrm{M}$ Ant A for 20 min caused a significant decrease in mitochondrial size in park ${ }^{25}$ mutant cells (Fig. $8 C$ ). However, mitochondrial engulfment into autophagosomes was still inhibited in park $k^{25}$ mutant neurons; the frequency of AVs containing mitochondrial signal from park ${ }^{25}$ mutants was reduced to $28 \%$ of control levels in cell bodies and $49 \%$ in axons (Fig. $8 A, B$ ). In addition, we tested the role of Parkin in mitochondrial colocalization with AVs under conditions of experimental autophagic induction. Cells were treated with $100 \mu \mathrm{M} \mathrm{H}_{2} \mathrm{O}_{2}$, which induces autophagy through oxidative stress (Court and Coleman, 2012), and should promote mitophagy. Again, mitochondrial size was significantly decreased in both control and park ${ }^{25}$ mutant cells by $\mathrm{H}_{2} \mathrm{O}_{2}$ treatment for $1 \mathrm{~h}$ (Fig. $8 \mathrm{C}$ ). However, even under conditions of oxidative stress, we saw the inhibition of mitochondrial engulfment in park ${ }^{25}$ mutant cells. In $\mathrm{H}_{2} \mathrm{O}_{2}$-treated cells, the frequency of AVs colocalizing with mitochondria was reduced to $18 \%$ of control levels in cell bodies and to $39 \%$ in axons (Fig. $8 A, B)$. Our results suggest that Parkin regulates mitophagy in vitro independently of the modulation of mitochondrial size. Furthermore, since Ant A and $\mathrm{H}_{2} \mathrm{O}_{2}$ treatments cause the fragmentation of mitochondria (Fig. $8 \mathrm{C}$ ) but no increase in AVs (data not shown), we also conclude that Parkin is dispensable for the remodeling of depolarized mitochondria.

Since we observed Parkin regulation of autophagosomal engulfment of mitochondria in vitro, we also asked whether axonal mitochondria in park ${ }^{25}$ mutant cells display normal membrane potential. The intensity ratio of TMRM staining showed that park ${ }^{25}$ mutant neurons indeed show diminished axonal $\Delta \Psi_{\mathrm{m}}$ (Fig. 9A,B). Consistent with previous analysis, axonal mitochondria were also elongated in park ${ }^{25}$ mutant cells (Fig. 9C). Thus, we regressed mitochondrial $\Delta \Psi_{\mathrm{m}}$ against their lengths to determine whether neurons in vitro display a key predicted consequence of Parkin-mediated QC: the accumulation of longer, less polarized mitochondria in the absence of Parkin. We found that park $k^{25}$ motor axons contained a small outlying population of longer mitochondria, and those mitochondria generally displayed diminished $\Delta \Psi_{\mathrm{m}}$ (Fig. $9 D, E$ ). Thus, as previously reported, we too find a significant in vitro role for Parkin in regulating axonal mitochondrial morphology and metabolic state; however, we do not observe this mechanism in the same neurons studied in vivo.

\section{Discussion}

It seems likely that the cellular maintenance and long-term survival of neurons require adequate mitochondrial QC. Because neurons are highly compartmentalized cells, with structurally and functionally distinct somatodendritic and axonal compartments, it is important to understand not just whether and how dysfunctional mitochondria are turned over, but also where this occurs. In cells in vitro, the PINK1/Parkin pathway has been proposed both to target dysfunctional mitochondria for turnover and, in a related fashion, to regulate mitochondrial transport and dynamics. We have tested these hypotheses about Parkin- dependent mitochondrial QC in the Drosophila nervous system both in vivo and in vitro, and show here the following: (1) Parkindeficient animals have dramatically reduced numbers of axonal mitochondria, but that the status and transport of these organelles remain normal; (2) the in vivo mitochondrial fission-fusion balance is regulated in a Parkin-dependent but somatically restricted manner; and (3) in vivo, mitophagy is rare in motor neurons and absent from their axons, but is both readily apparent and Parkin dependent in the same neurons in vitro.

Axonal transport of mitochondria has long been held to be intimately related to other organelle functions such as fissionfusion, metabolism, and turnover (Miller and Sheetz, 2004; Baloh et al., 2007; Pathak et al., 2010; Arduíno et al., 2012; Saxton and Hollenbeck, 2012). The PINK1/Parkin pathway has been proposed to coordinate mitochondrial motility with organelle turnover by specifically dissociating kinesin, the microtubulebased anterograde motor, from the organelle surface through degradation of its linker protein Miro (Wang et al., 2011). This model, however, does not explain how or why the modulation of the pathway disrupts mitochondrial movement in both the anterograde and retrograde directions. Since disruptions targeted to one direction of microtubule-based axonal transport often alter movement in both directions (Pilling et al., 2006; Park et al., 2009; Russo et al., 2009), it seems likely that the regulation of transport includes intricate coupling of both motors, kinesin and dynein. In any case, contrary to the proposed model of PINK1/ Parkin dependent mitochondrial arrest, here we observed nearly unchanged mitochondrial motility behavior in vivo when Parkin expression was perturbed. Thus, although we found that Parkin deletion dramatically reduced mitochondrial flux in motor axons in vivo (Fig. 1C), this was derived almost entirely from a reduction in mitochondrial density to one-half that of normal axons (Figs. 2,3), with only very modest, directionally balanced changes in motility. This is the opposite of the result expected for Parkin deletion, if this pathway were driving clearance of senescent mitochondria from the axon. It is, however, consistent with our previous demonstration that manipulation of PINK1 also fails to affect mitochondrial arrest (Devireddy et al., 2015). Since the PINK1/Parkin pathway is proposed not only to target damaged mitochondria for local turnover (Narendra et al., 2008, 2010; Matsuda et al., 2010; Ashrafi et al., 2014) but also to inhibit organelle fusion (Deng et al., 2008; Poole et al., 2010; Ziviani et al., 2010), we also expected Parkin deletion to produce elongated and metabolically compromised mitochondria in vivo. Surprisingly, our quantitative analyses demonstrated that mutant animals preserve both normal mitochondrial morphology and $\Delta \Psi_{\mathrm{m}}$ in their axons and NMJs (Fig. 4). On the other hand, in the cell bodies of Parkin-deficient motor neurons in vivo, organelle morphology was dramatically altered (Fig. 5), which suggests a compartmentalized in vivo role for Parkin in mitochondrial QC.

What could be restricting Parkin-dependent mitochondrial QC to cell bodies in vivo? One possibility is the dynamic interplay with other organelles, such as endoplasmic reticulum (ER). ER tubules form membrane contact sites with a number of other organelles (Rowland and Voeltz, 2012; Rowland et al., 2014), and such contacts regulate diverse neuronal functions, including motor protein-dependent organelle transport and neurite outgrowth (Raiborg et al., 2015). Recently, it has been proposed that ER tubules also play a regulatory role in mitochondrial division by contacting mitochondria and causing their constriction before the recruitment of the mitochondrial fission protein Drp1 (Friedman et al., 2011). Although ER elements are found in the axon and contribute to the transport and distribution of axonal 
A
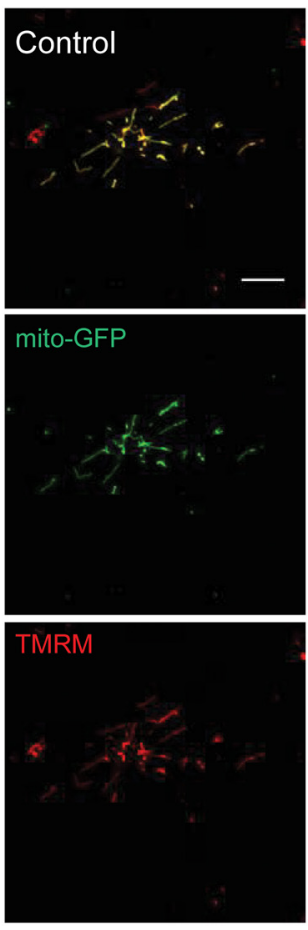
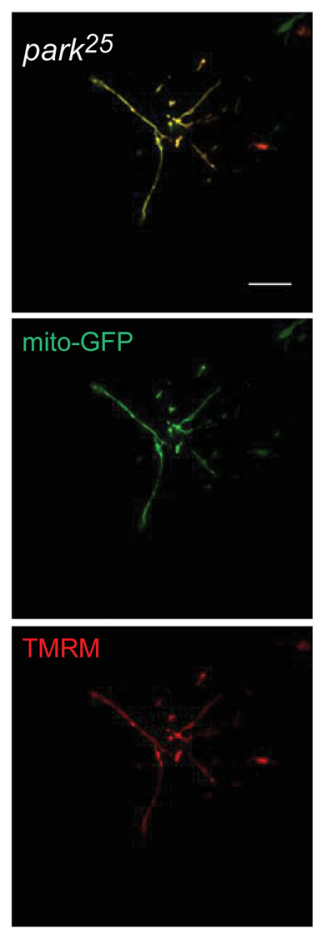

B

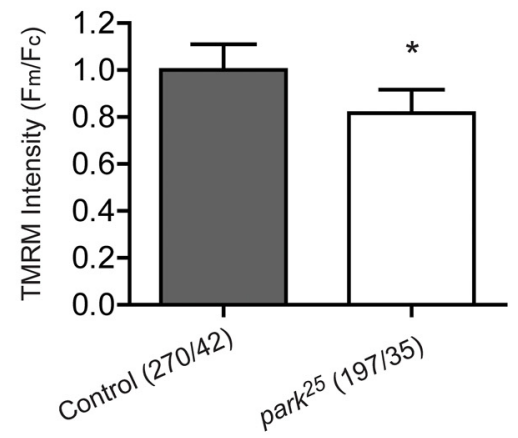

C

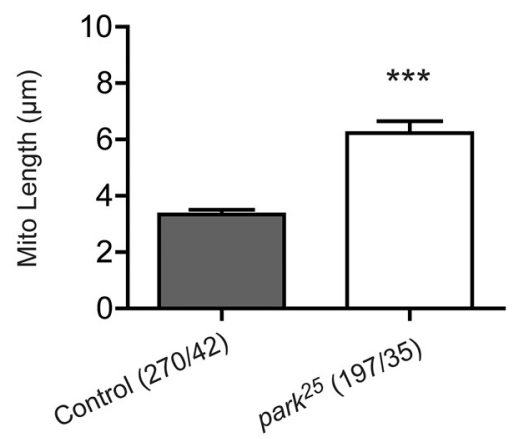

D

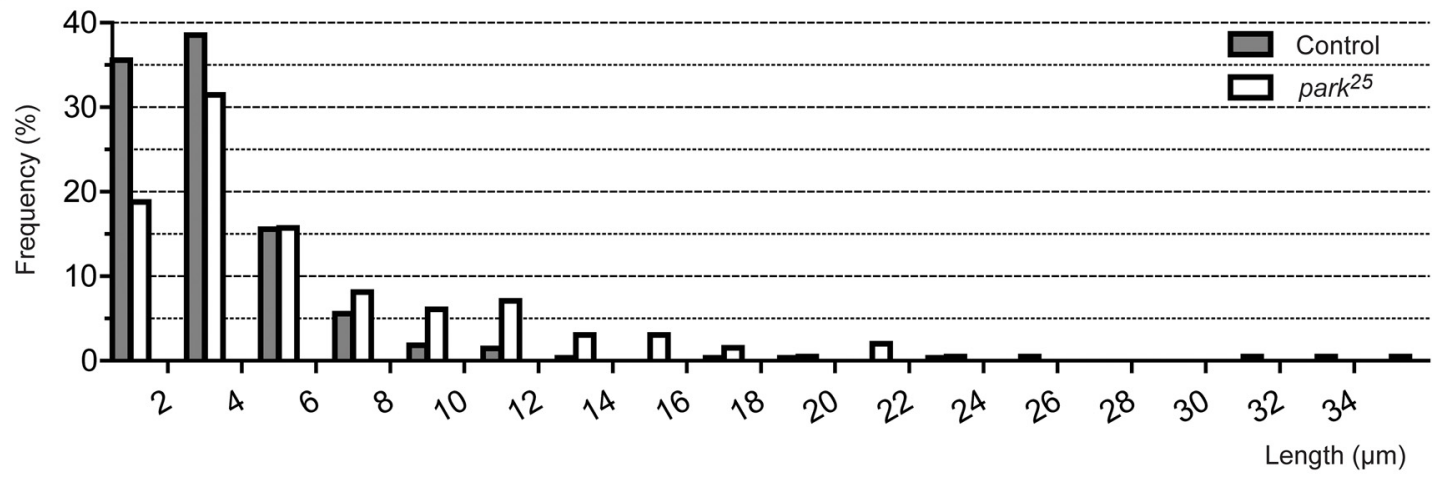

$\mathbf{E}$

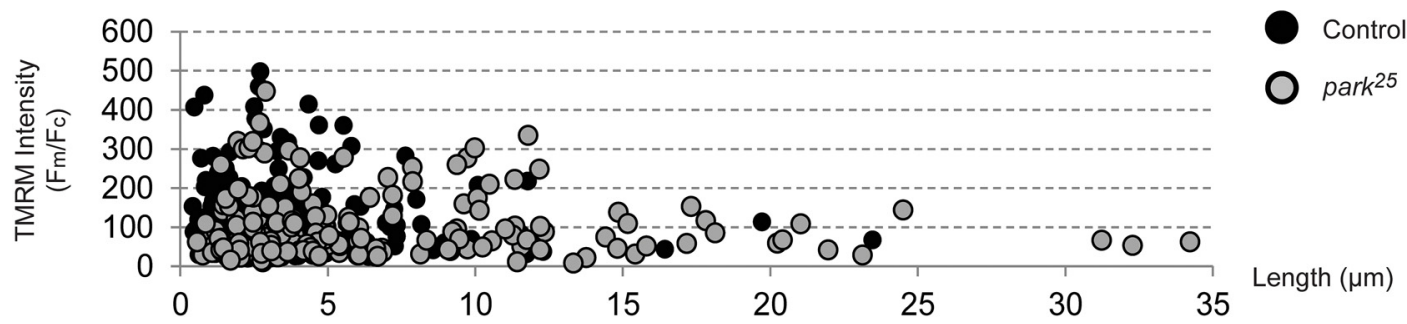

Figure 9. Parkin perturbation produced elongated mitochondria with diminished mitochondrial $\Delta \Psi_{\mathrm{m}}$ in vitro. $A$, Representative images of motor neurons stained with TMRM in vitro after $96 \mathrm{~h}$ in culture. Scale bars, $10 \mu \mathrm{m} . \boldsymbol{B}$, Mean $\mathrm{F}_{\mathrm{m}} / \mathrm{F}_{\mathrm{c}}$ intensity ratio of TMRM from cultured neurons of different parkin genotypes. $C$, Mean length of axonal mitochondria in vitro. $\boldsymbol{D}$, Length distribution of axonal mitochondria in vitro. $E$, Relationship between mitochondrial $\Delta \Psi_{\mathrm{m}}$ and length in axons in vitro. Correlation coefficients: control $\left(R^{2}=0.0049\right)$ and park $k^{25}\left(R^{2}=0.0004\right)$. park $k^{25}$ mutant neurons in vitro show decreased $\Delta \Psi_{\mathrm{m}}$ and elongated axonal mitochondria. $(n / n)$, Number of mitochondria/number of cells. Error bars indicate the mean \pm SEM. Significance is determined by Student's $t$ test $\left(\boldsymbol{B}, \boldsymbol{C} ;{ }^{*} p<0.05\right.$ and $\left.{ }^{* * *} p<0.001\right)$ or by two-sample Kolmogorov-Smirnov test $(\boldsymbol{D} ; \mathrm{K}-\mathrm{S}$ statistics show significant difference; $D=0.2107>$ critical $D=0.1274$ with a corresponding $p<0.001)$.

cargos (González and Couve, 2014), ER tubules apparently maintain their contact sites with other organelles independent of microtubules (Rowland et al., 2014), and their major network is located in the cell body (Berridge, 1998; Verkhratsky and Petersen, 1998). Thus, it is plausible that the role of ER in mitochon- drial morphology restricts the compartmental localization of the defects caused by Parkin perturbation in vivo. In addition, if the somatic compartment is the main site of mitochondrial turnover (Safiulina and Kaasik, 2013), then the entry of mitochondria into the axon from the cell body, and perhaps their subsequent return, 
are critically important in regulating the neuronal mitochondrial life cycle. In this context, we propose that mature neurons possess a QC barrier between the cell body and the axon that restricts axonal entry to those mitochondria of appropriate size, morphology, and metabolic status. This proposal is consistent with recent evidence that the preaxonal exclusion zone in the hillock provides a cytoplasmic boundary for the entry of vesicles into the axonal domain (Farías et al., 2015). Thus, the disruption of the fissionfusion balance in cell bodies by Parkin perturbation, which caused abnormal, interconnected mitochondria in the cell body, may physically disrupt the "launching process" of dysfunctional organelles into the axon.

If new mitochondria to supply the axon are generated largely in the cell body in vivo, then the importance of axonal transport looms even larger, especially anterograde movement. Indeed, it is notable that the same reduced-axonal-density phenotype that we observed has previously been reported in motor axons of Drosophila SNs with disrupted motor protein expression (Pilling et al., 2006). In that study, it was remarkable that only kinesin heavy chain mutants displayed reduced mitochondrial density in SNs, while mutations in dynein retained the normal compliment of axonal mitochondria. Furthermore, our previous report demonstrated that Parkin selectively restored the abnormal mitochondria of PINK1 mutants only in cell bodies, not in axons (Devireddy et al., 2015). In addition, it is noteworthy that a previous study (Cai et al., 2012) of cultured cortical neurons in vitro shows the restricted Parkin-mediated mitophagy only in the somatodendritic compartment. Together, these findings suggest a compartmentalized in vivo role of PINK1/Parkin in neuronal mitochondrial turnover and provide further evidence that the mitochondrial supply from the cell body is critically important for preserving organelle numbers and distribution in axons.

We did observe the morphological remodeling of mitochondria under stress conditions both in vivo and in vitro: starvation in vivo (Fig. 6A), as well as Ant $\mathrm{A}$ and $\mathrm{H}_{2} \mathrm{O}_{2}$ treatments in vitro (Fig. $8 C$ ) both produced fragmented mitochondria, even in the absence of Parkin. It is possible that mitochondrial fission is regulated independently of Parkin and/or upregulated by stress conditions. However, we observed several dramatic distinctions in the mitochondrial life cycle between motor neurons in vivo and in vitro. First, we rarely observed AVs that colocalized with mitochondria anywhere in motor neurons in vivo, whereas Parkindependent mitophagy was obvious in the same neurons in vitro. Second, axonal mitochondria under Parkin perturbation were elongated and displayed diminished $\Delta \Psi_{\mathrm{m}}$ in motor neurons in vitro, but had virtually normal status in vivo. Thus, while our observations from in vitro neurons supported the current models for the role of Parkin in axonal mitochondrial QC, targeting senescent mitochondria and promoting mitochondrial fission, data from the same neurons in vivo expressly did not.

What could explain these differences? One possibility is that fully connected neurons in vivo have established a physical filter for mitochondria between the cell body and axon that remains less developed in vitro. In support of this idea, we noted that the most axonal mitochondria in vivo were morphologically nearly uniform and approximately two times shorter than axonal mitochondria in cultured neurons (Figs. $4 A, 9 C$ ). In addition, axonal mitochondria in vitro often extended continuously from the cell body into the axon (Fig. $8 A$ ), a feature that is rarely, if ever, seen in vivo, suggesting that the filter between the cell body and axon is absent or more permissive in cultured neurons. If mitochondrial entry into the axon is more restricted in vivo than in vitro, it could also explain the greatly downregulated axonal mitophagy that we observed in vivo. It is also important to note that mature neurons in vivo are functionally associated with surrounding cells and tissues. Recent evidence from mouse optic nerve head in vivo (Davis et al., 2014) shows the extrusion and transcellular degradation of axonal mitochondria, "transmitophagy," a process that could provide a different avenue for mitochondrial turnover in the periphery, and could contribute to the lack of axonal mitophagy observed in vivo. It is also likely that neurons in vitro, subjected to hyperoxic conditions, are more susceptible to environmental stress. Thus, they may upregulate their mechanisms for mitochondrial clearance.

In summary, comparing Drosophila motor neurons in vivo and in vitro, we find a dramatic difference that indicates a highly compartmentalized in vivo role for Parkin in mitochondrial QC. Unlike in vitro conditions, the in vivo role of Parkin does not appear obviously related to mitophagy, and is restricted to the cell body where it seems to modulate mitochondrial fission-fusion balance and, indirectly, the mitochondrial composition of the axon. Our results re-emphasize the intricate interdependence of mitochondrial dynamics and axonal transport, and suggest that neuronal mitochondrial QC occurs in the cell body, and that a critical step is the initial access of mitochondria to the axon after organelle biogenesis.

\section{References}

Alirezaei M, Kemball CC, Flynn CT, Wood MR, Whitton JL, Kiosses WB (2010) Short-term fasting induces profound neuronal autophagy. Autophagy 6:702-710. CrossRef Medline

Amiri M, Hollenbeck PJ (2008) Mitochondrial biogenesis in the axons of vertebrate peripheral neurons. Dev Neurobiol 68:1348-1361. CrossRef Medline

Arduíno DM, Esteves AR, Cortes L, Silva DF, Patel B, Grazina M, Swerdlow RH, Oliveira CR, Cardoso SM (2012) Mitochondrial metabolism in Parkinson's disease impairs quality control autophagy by hampering microtubule-dependent traffic. Hum Mol Genet 21:4680-4702. CrossRef Medline

Ashrafi G, Schlehe JS, LaVoie MJ, Schwarz TL (2014) Mitophagy of damaged mitochondria occurs locally in distal neuronal axons and requires PINK1 and Parkin. J Cell Biol 206:655-670. CrossRef Medline

Bai J, Sepp KJ, Perrimon N (2009) Culture of Drosophila primary cells dissociated from gastrula embryos and their use in RNAi screening. Nat Protoc 4:1502-1512. CrossRef Medline

Baloh RH, Schmidt RE, Pestronk A, Milbrandt J (2007) Altered axonal mitochondrial transport in the pathogenesis of Charcot-Marie-Tooth disease from mitofusin 2 mutations. J Neurosci 27:422-430. CrossRef Medline

Barth JM, Szabad J, Hafen E, Köhler K (2011) Autophagy in Drosophila ovaries is induced by starvation and is required for oogenesis. Cell Death Differ 18:915-924. CrossRef Medline

Berridge MJ (1998) Neuronal calcium signaling. Neuron 21:13-26. CrossRef Medline

Cagalinec M, Safiulina D, Liiv M, Liiv J, Choubey V, Wareski P, Veksler V, Kaasik A (2013) Principles of the mitochondrial fusion and fission cycle in neurons. J Cell Sci 126:2187-2197. CrossRef Medline

Cai Q, Zakaria HM, Simone A, Sheng ZH (2012) Spatial parkin translocation and degradation of damaged mitochondria via mitophagy in live cortical neurons. Curr Biol 22:545-552. CrossRef Medline

Chan DC (2006) Mitochondrial fusion and fission in mammals. Annu Rev Cell Dev Biol 22:79-99. CrossRef Medline

Chen H, Chan DC (2009) Mitochondrial dynamics-fusion, fission, movement, and mitophagy - in neurodegenerative diseases. Hum Mol Genet 18:R169-R176. CrossRef Medline

Chen X, Kondo K, Motoki K, Homma H, Okazawa H (2015) Fasting activates macroautophagy in neurons of Alzheimer's disease mouse model but is insufficient to degrade amyloid-beta. Sci Rep 5:12115. CrossRef Medline

Court FA, Coleman MP (2012) Mitochondria as a central sensor for axonal degenerative stimuli. Trends Neurosci 35:364-372. CrossRef Medline

Davis AF, Clayton DA (1996) In situ localization of mitochondrial DNA 
replication in intact mammalian cells. J Cell Biol 135:883-893. CrossRef Medline

Davis CH, Kim KY, Bushong EA, Mills EA, Boassa D, Shih T, Kinebuchi M, Phan S, Zhou Y, Bihlmeyer NA, Nguyen JV, Jin Y, Ellisman MH, MarshArmstrong N (2014) Transcellular degradation of axonal mitochondria. Proc Natl Acad Sci U S A 111:9633-9638. CrossRef Medline

Deinhardt K, Salinas S, Verastegui C, Watson R, Worth D, Hanrahan S, Bucci C, Schiavo G (2006) Rab5 and Rab7 control endocytic sorting along the axonal retrograde transport pathway. Neuron 52:293-305. CrossRef Medline

Deng H, Dodson MW, Huang H, Guo M (2008) The Parkinson's disease genes pink1 and parkin promote mitochondrial fission and/or inhibit fusion in Drosophila. Proc Natl Acad Sci U S A 105:14503-14508. CrossRef Medline

Devireddy S, Sung H, Liao PC, Garland-Kuntz E, Hollenbeck PJ (2014) Analysis of mitochondrial traffic in Drosophila. Methods Enzymol 547: 131-150. CrossRef Medline

Devireddy S, Liu A, Lampe T, Hollenbeck PJ (2015) The organization of mitochondrial quality control and life cycle in the nervous system in vivo in the absence of PINK1. J Neurosci 35:9391-9401. CrossRef Medline

Fang Y, Soares L, Teng X, Geary M, Bonini NM (2012) A novel Drosophila model of nerve injury reveals an essential role of Nmnat in maintaining axonal integrity. Curr Biol 22:590-595. CrossRef Medline

Fang Y, Soares L, Bonini NM (2013) Design and implementation of in vivo imaging of neural injury responses in the adult Drosophila wing. Nat Protoc 8:810-819. CrossRef Medline

Farías GG, Guardia CM, Britt DJ, Guo X, Bonifacino JS (2015) Sorting of dendritic and axonal vesicles at the pre-axonal exclusion zone. Cell Rep 13:1221-1232. CrossRef Medline

Friedman JR, Lackner LL, West M, DiBenedetto JR, Nunnari J, Voeltz GK (2011) ER tubules mark sites of mitochondrial division. Science 334: 358-362. CrossRef Medline

Ghosh D, Walton JL, Roepe PD, Sinai AP (2012) Autophagy is a cell death mechanism in Toxoplasma gondii. Cell Microbiol 14:589-607. CrossRef Medline

Glater EE, Megeath LJ, Stowers RS, Schwarz TL (2006) Axonal transport of mitochondria requires milton to recruit kinesin heavy chain and is light chain independent. J Cell Biol 173:545-557. CrossRef Medline

Gomes LC, Di Benedetto G, Scorrano L (2011) During autophagy mitochondria elongate, are spared from degradation and sustain cell viability. Nat Cell Biol 13:589-598. CrossRef Medline

González C, Couve A (2014) The axonal endoplasmic reticulum and protein trafficking: cellular bootlegging south of the soma. Semin Cell Dev Biol 27:23-31. CrossRef Medline

Greene JC, Whitworth AJ, Kuo I, Andrews LA, Feany MB, Pallanck LJ (2003) Mitochondrial pathology and apoptotic muscle degeneration in Drosophila parkin mutants. Proc Natl Acad Sci U S A 100:4078-4083. CrossRef Medline

Guo X, Macleod GT, Wellington A, Hu F, Panchumarthi S, Schoenfield M, Marin L, Charlton MP, Atwood HL, Zinsmaier KE (2005) The GTPase dMiro is required for axonal transport of mitochondria to Drosophila synapses. Neuron 47:379-393. CrossRef Medline

Hirokawa N, Niwa S, Tanaka Y (2010) Molecular motors in neurons: transport mechanisms and roles in brain function, development, and disease. Neuron 68:610-638. CrossRef Medline

Lazarou M, Sliter DA, Kane LA, Sarraf SA, Wang C, Burman JL, Sideris DP, Fogel AI, Youle RJ (2015) The ubiquitin kinase PINK1 recruits autophagy receptors to induce mitophagy. Nature 524:309-314. CrossRef Medline

Liu S, Sawada T, Lee S, Yu W, Silverio G, Alapatt P, Millan I, Shen A, Saxton W, Kanao T, Takahashi R, Hattori N, Imai Y, Lu B (2012) Parkinson's disease-associated kinase PINK1 regulates Miro protein level and axonal transport of mitochondria. PLoS Genet 8:e1002537. CrossRef Medline

Louie K, Russo GJ, Salkoff DB, Wellington A, Zinsmaier KE (2008) Effects of imaging conditions on mitochondrial transport and length in larval motor axons of Drosophila. Comp Biochem Physiol A Mol Integr Physiol 151:159-172. CrossRef Medline

Maday S, Holzbaur EL (2014) Autophagosome biogenesis in primary neurons follows an ordered and spatially regulated pathway. Dev Cell 30:7185. CrossRef Medline

Maday S, Wallace KE, Holzbaur EL (2012) Autophagosomes initiate distally and mature during transport toward the cell soma in primary neurons. J Cell Biol 196:407-417. CrossRef Medline

Matsuda N, Sato S, Shiba K, Okatsu K, Saisho K, Gautier CA, Sou YS, Saiki S, Kawajiri S, Sato F, Kimura M, Komatsu M, Hattori N, Tanaka K (2010) PINK1 stabilized by mitochondrial depolarization recruits Parkin to damaged mitochondria and activates latent Parkin for mitophagy. J Cell Biol 189:211-221. CrossRef Medline

Miller KE, Sheetz MP (2004) Axonal mitochondrial transport and potential are correlated. J Cell Sci 117:2791-2804. CrossRef Medline

Misko A, Jiang S, Wegorzewska I, Milbrandt J, Baloh RH (2010) Mitofusin 2 is necessary for transport of axonal mitochondria and interacts with the Miro/Milton complex. J Neurosci 30:4232-4240. CrossRef Medline

Moughamian AJ, Holzbaur EL (2012) Dynactin is required for transport initiation from the distal axon. Neuron 74:331-343. CrossRef Medline

Narendra D, Tanaka A, Suen DF, Youle RJ (2008) Parkin is recruited selectively to impaired mitochondria and promotes their autophagy. J Cell Biol 183:795-803. CrossRef Medline

Narendra DP, Jin SM, Tanaka A, Suen DF, Gautier CA, Shen J, Cookson MR, Youle RJ (2010) PINK1 is selectively stabilized on impaired mitochondria to activate Parkin. PLoS Biol 8:e1000298. CrossRef Medline

Okamoto K, Shaw JM (2005) Mitochondrial morphology and dynamics in yeast and multicellular eukaryotes. Annu Rev Genet 39:503-536. CrossRef Medline

Park JJ, Koshimizu H, Loh YP (2009) Biogenesis and transport of secretory granules to release site in neuroendocrine cells. J Mol Neurosci 37:151159. CrossRef Medline

Pathak D, Sepp KJ, Hollenbeck PJ (2010) Evidence that myosin activity opposes microtubule-based axonal transport of mitochondria. J Neurosci 30:8984-8992. CrossRef Medline

Pilling AD, Horiuchi D, Lively CM, Saxton WM (2006) Kinesin-1 and Dynein are the primary motors for fast transport of mitochondria in Drosophila motor axons. Mol Biol Cell 17:2057-2068. CrossRef Medline

Poole AC, Thomas RE, Andrews LA, McBride HM, Whitworth AJ, Pallanck LJ (2008) The PINK1/Parkin pathway regulates mitochondrial morphology. Proc Natl Acad Sci U S A 105:1638-1643. CrossRef Medline

Poole AC, Thomas RE, Yu S, Vincow ES, Pallanck L (2010) The mitochondrial fusion-promoting factor mitofusin is a substrate of the PINK1/parkin pathway. PLoS One 5:e10054. CrossRef Medline

Raiborg C, Wenzel EM, Pedersen NM, Olsvik H, Schink KO, Schultz SW, Vietri M, Nisi V, Bucci C, Brech A, Johansen T, Stenmark H (2015) Repeated ER-endosome contacts promote endosome translocation and neurite outgrowth. Nature 520:234-238. CrossRef Medline

Rambold AS, Kostelecky B, Elia N, Lippincott-Schwartz J (2011) Tubular network formation protects mitochondria from autophagosomal degradation during nutrient starvation. Proc Natl Acad Sci U S A 108:1019010195. CrossRef Medline

Reis K, Fransson A, Aspenström P (2009) The Miro GTPases: at the heart of the mitochondrial transport machinery. FEBS Lett 583:1391-1398. CrossRef Medline

Rowland AA, Voeltz GK (2012) Endoplasmic reticulum-mitochondria contacts: function of the junction. Nat Rev Mol Cell Biol 13:607-625. CrossRef Medline

Rowland AA, Chitwood PJ, Phillips MJ, Voeltz GK (2014) ER contact sites define the position and timing of endosome fission. Cell 159:1027-1041. CrossRef Medline

Rugarli EI, Langer T (2012) Mitochondrial quality control: a matter of life and death for neurons. EMBO J 31:1336-1349. CrossRef Medline

Russo GJ, Louie K, Wellington A, Macleod GT, Hu F, Panchumarthi S, Zinsmaier KE (2009) Drosophila Miro is required for both anterograde and retrograde axonal mitochondrial transport. J Neurosci 29:5443-5455. CrossRef Medline

Safiulina D, Kaasik A (2013) Energetic and dynamic: how mitochondria meet neuronal energy demands. PLoS Biol 11:e1001755. CrossRef Medline

Saxton WM, Hollenbeck PJ (2012) The axonal transport of mitochondria. J Cell Sci 125:2095-2104. CrossRef Medline

Shidara Y, Hollenbeck PJ (2010) Defects in mitochondrial axonal transport and membrane potential without increased reactive oxygen species production in a Drosophila model of Friedreich ataxia. J Neurosci 30:1136911378. CrossRef Medline

Slater EC (1973) The mechanism of action of the respiratory inhibitor, antimycin. Biochim Biophys Acta 301:129-154. CrossRef Medline 
Song Z, Ghochani M, McCaffery JM, Frey TG, Chan DC (2009) Mitofusins and OPA1 mediate sequential steps in mitochondrial membrane fusion. Mol Biol Cell 20:3525-3532. CrossRef Medline

Stowers RS, Megeath LJ, Górska-Andrzejak J, Meinertzhagen IA, Schwarz TL (2002) Axonal transport of mitochondria to synapses depends on milton, a novel Drosophila protein. Neuron 36:1063-1077. CrossRef Medline

Verburg J, Hollenbeck PJ (2008) Mitochondrial membrane potential in axons increases with local nerve growth factor or semaphorin signaling. J Neurosci 28:8306-8315. CrossRef Medline

Verkhratsky AJ, Petersen OH (1998) Neuronal calcium stores. Cell Calcium 24:333-343. CrossRef Medline

Verstreken P, Ly CV, Venken KJ, Koh TW, Zhou Y, Bellen HJ (2005) Syn- aptic mitochondria are critical for mobilization of reserve pool vesicles at Drosophila neuromuscular junctions. Neuron 47:365-378. CrossRef Medline

Wang X, Winter D, Ashrafi G, Schlehe J, Wong YL, Selkoe D, Rice S, Steen J, LaVoie MJ, Schwarz TL (2011) PINK1 and Parkin target Miro for phosphorylation and degradation to arrest mitochondrial motility. Cell 147: 893-906. CrossRef Medline

Yu Y, Lee HC, Chen KC, Suhan J, Qiu M, Ba Q, Yang G (2016) Inner membrane fusion mediates spatial distribution of axonal mitochondria. Sci Rep 6:18981. CrossRef Medline

Ziviani E, Tao RN, Whitworth AJ (2010) Drosophila parkin requires PINK1 for mitochondrial translocation and ubiquitinates mitofusin. Proc Natl Acad Sci U S A 107:5018-5023. CrossRef Medline 